\title{
Circular RNA circ_0001421 contributes to colony formation, migration, invasion and glycolysis of non-small cell lung cancer via the miR-409-3p/TMEM14A axis
}

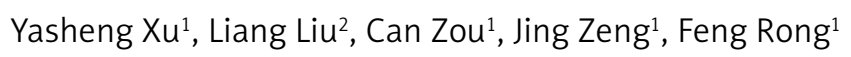

\author{
${ }^{1}$ Department of Respiratory, Xiantao First People's Hospital Affiliated to Changjiang \\ University, Xiantao, Hubei, China \\ 2Department of Oncology, Xiantao First People's Hospital Affiliated to Changjiang \\ University, Xiantao, Hubei, China
}

Submitted: 26.12 .2019

Accepted: 10.04 .2020

Arch Med Sci

DOI: https://doi.org/10.5114/aoms/120369

Copyright $\odot 2020$ Termedia \& Banach

\section{Abstract}

Introduction: Accumulating evidence testifies to the considerably significant roles of circular RNAs (circRNAs) in non-small cell lung cancer (NSCLC). This report describes the exploration of the molecular mechanism of circRNA_0001421 (circ_0001421) in NSCLC.

Material and methods: The relative levels of circ_0001421, microRNA-409-3p (miR-409-3p) and transmembrane protein 14A (TMEM14A) were assayed through quantitative real-time polymerase chain reaction (qRT-PCR). Cell colony formation ability was detected by colony formation assay. Transwell assay was exploited for assessing cell migration and invasion. Glycolysis was evaluated via ECAR measurement, glucose consumption, lactate production and protein detection. The protein levels were examined using Western blot. The target combination between miR-409-3p and circ_0001421 or TMEM14A was analyzed by dual-luciferase reporter assay. Xenotransplantation assay was applied for estimating the effect of circ_0001421 on NSCLC in 10 mice. Results: Circ_0001421 was up-regulated in NSCLC tissues and cells. Down-regulation of circ_0001421 suppressed colony formation, migration, invasion and glycolysis of NSCLC cells. Circ_0001421 could sponge microRNA-409-3p (miR-409-3p) and miR-409-3p inhibition relieved the effects of circ 0001421 knockdown on NSCLC cells. MiR-409-3p targeted transmembrane protein 14A (TMEM14A) and circ_0001421 modulated TMEM14A expression via targeting miR-409-3p. Overexpression of miR-409-3p suppressed NSCLC progression by inhibiting TMEM14A. Circ_0001421 depression restrained tumor growth of NSCLC by the miR-409-3p/TMEM14A axis in vivo.

Conclusions: Circ_0001421 facilitated the development of NSCLC via the regulation of the miR-409-3p/TMEM14A axis. Circ_0001421 may be a promising therapeutic target in NSCLC.

Key words: circ_0001421, non-small cell lung cancer, miR-409-3p, TMEM14A.

\section{Introduction}

One of the leading causes of cancer-related death is lung cancer, in which non-small cell lung cancer (NSCLC) accounts for approximately $85 \%$ of lung-cancer-caused mortality $[1,2]$. The NSCLC patients can obtain re-

\author{
Corresponding author: \\ Feng Rong \\ Department of Respiratory \\ Xiantao First People's \\ Hospital Affiliated to \\ Changjiang University \\ No. 29 Middle Section of \\ Mianzhou Avenue \\ Nancheng New District, \\ Xiantao 433000, Hubei, \\ China \\ Phone: $+86-15871888698$ \\ E-mail: reqkfi@163.com
}


mission with modern therapies, such as radio-chemotherapy, immunotherapy, epidermal growth factor receptor tyrosine kinase inhibitors (EGFR TKIs) therapy and ALK rearrangements [2-5]. But the 5 -year survival rate for patients with metastatic NSCLC remains extremely poor [6]. Some studies have indicated that non-coding RNAs (ncRNAs) could act as regulators in the development and as biomarkers in the treatment of NSCLC $[7,8]$. Hence, investigation of the mechanism of more novel ncRNAs in NSCLC is urgently needed.

Circular RNAs (circRNAs), a class of highly conserved ncRNAs, lack 5' and 3' polarity. The covalently closed-loop structures endow circRNAs with low degradation and high stability $[9,10]$. The involvement of circRNAs in human cancers has been gradually recognized. Guan et al. asserted that circPUM1 was conductive to the tumorigenesis of ovarian cancer acting as sponges of miR615-5p and miR-6753-5p [11]. Wang et al. discovered that the up-regulation of hsa_circ_0000517 could serve as a prognostic marker in hepatocellular carcinoma [12]. Liu et al. reported that hsa circ_103809 expedited the progression of lung cancer via the miR-4302/ZNF121/MYC axis [13]. A recent report showed abnormally high expression of circ_0001421 in NSCLC tissues [14], while the role of circ_0001421 in NSCLC is unknown.

As other frequent ncRNAs, miRNAs usually participate in varied biological behaviors and the development of tumors as pivotal regulators $[15,16]$. MiR-221 was shown to function as a tumor promoter in NSCLC by targeting TIMP2 [17]. Ma et al. proved the antitumor role of miR-223 in NSCLC via the EGFR-mediated PI3K/AKT signal pathway [18]. Song et al. reported that miR-409 restricted NSCLC progression by regulating SPIN1 [19]. Also miR-409-3p was found to be involved in the NSCLC progression regulated by long nCRNA (IncRNA) ZEB1-AS1 [20]. Herein, we intended to explore the influence of miR-409-3p on NSCLC and the relation with circ_0001421.

Transmembrane protein 14A (TMEM14A) belongs to the TMEM family, and plays an essential role in several cancers. For example, the knockdown of TMEM14A was manifested to decrease cell proliferation and invasion in ovarian cancer [21]. An et al. reported that TMEM14A was overexpressed and regulated by the circ_0003645/miR1179 axis in NSCLC [22]. Our study focused on the connection among circ_0001421, miR-409-3p and TMEM14A in the regulation of cell colony formation, migration, invasion and glycolysis of NSCLC.

\section{Material and methods}

\section{Tissues source and cell culture}

After obtaining the informed consent from NSCLC patients, a total of 38 pairs of NSCLC tissues and normal peritumor tissues were acquired at Xiantao First People's Hospital Affiliated to Changjiang University, followed by the preservation of samples in liquid nitrogen. These patients did not receive any other therapy prior to pneumonectomy. Our research was authorized by the Institute Review Ethics Committee of Xiantao First People's Hospital Affiliated to Changjiang University.

Human NSCLC cell lines (H1975 and A549) and the normal lung epithelial BEAS-2B cell line from American Type Culture Collection (ATCC, Manassas, VA, USA) were cultivated in the environment of $37^{\circ} \mathrm{C}, 5 \% \mathrm{CO}_{2}$ and $95 \%$ air. The culture medium consisted of the basic medium Roswell Park Memorial Institute-1640 (RPMI-1640; Life Technologies, Carlsbad, CA, USA), 10\% fetal bovine serum (FBS; Serapro, Naila, Germany) and 1\% penicillin-streptomycin (Gibco, Carlsbad, CA, USA).

\section{Transient transfection}

Small interfering RNA (siRNA) against circ 0001421 (si-circ 0001421), miR-409-3p mimic and inhibitor (miR-409-3p and anti-miR-409-3p) were synthesized by GENEWIZ (Suzhou, China). Vector construction was implemented by cloning the open reading frame (ORF) of TMEM14A into the pcDNA3.0 basic vector (Invitrogen, Carlsbad, CA, USA) to overexpress TMEM14A (TMEM14A). Transiently, these oligonucleotides or vector were transfected into NSCLC cells following the Lipofectamine 3000 (Invitrogen) protocol. Si-NC, miRNC, anti-miR-NC and vector were used as the negative controls for the above transfection.

\section{Quantitative real-time polymerase chain reaction}

The RNA extraction was executed using Trizol (Invitrogen), and $2 \mu \mathrm{g}$ RNA was used to synthesize complementary DNA (cDNA) with the PrimeScript RT Master Mix (Takara, Dalian, China), then quantitative real-time polymerase chain reaction (qRT-PCR) reaction was conducted using a Fast SYBR Green PCR kit (Applied Biosystems, Foster City, CA, USA). We used glyceraldehyde-3-phosphate dehydrogenase (GAPDH, for circ_0001421 and TMEM14A) and U6 (for miR-409-3p) as in ternal controls to normalize the expression levels. Primer sequences were as follows: circ 0001421 (forward: 5'-TGCCCAGAATGACAAGCATA-3' and reverse: 5'-TGAGCAGATGTTCCTGTTGC-3'); miR-4093p (forward: 5'-GGGGAATGTTGCTCGGTGA-3' and reverse: 5'-CAGTGCGTGTCGTGGAGT-3'); TMEM14A (forward: 5'-GTTTGGTTGCAGGTTTAAG-3' and reverse: 5'-AATATGCTCTGCCCATTAC-3'); GAPDH (forward: 5'-GTCTCCTCTGACTTCAACAGCG-3' and reverse: 5'-ACCACCCTGTTGCTGTAGCCAA-3'); U6 (for- 
ward: 5'-CTCGCTTCGGCAGCACA-3' and reverse: 5'-AACGCTTCACGAATTTGCGT-3'). Eventually, the data were exhibited as relative expression levels by the $2^{-\Delta \Delta \mathrm{Ct}}$ method [23].

\section{Localization analysis}

The supernatant from centrifugal cell lysate was considered as the cytoplasmic fraction and the pellet was the nucleus fraction. The PARIS Kit (Invitrogen) was applied for isolating RNA from the nuclear and cytoplasmic fractions, referring to the user's guideline. The expression levels of circ_0001421, GAPDH and U6 were detected by qRT-PCR using two RNA as samples. GAPDH and U6 were separately used as the positive controls of cytoplasmic and nuclear fractions.

\section{Colony formation assay}

Transfected NSCLC cells were plated into 6-well plates with 1000 cells per well. Following inoculation about 2 weeks, macroscopic colonies were fixed using 4\% paraformaldehyde and dyed with crystal violet (Sangon, Shanghai, China). After washing with phosphate buffer solution (PBS; Corning, New York, NY, USA), the counting of colonies was performed with a microscope.

\section{Detection of migration and invasion}

Transwell assay was administered to assess cell migration and invasion abilities. Concisely, a $100 \mu \mathrm{l}$ cell suspension was pipetted into the upper chamber of a transwell chamber (Corning) and $600 \mu \mathrm{l}$ of culture medium was added to the lower chamber, incubating for $24 \mathrm{~h}$. After the respective fixing and staining with $4 \%$ paraformaldehyde and crystal violet (Sangon), the migrated cells were counted under a microscope. But in the detection of invasion, the upper chamber must be coated with Matrigel (Corning) before cell seeding, and invaded cells were calculated.

\section{Measurement of the extracellular acidification rate}

The Seahorse Extracellular Flux Analyzer XF96 (Seahorse Bioscience, Billerica, MA, USA) was used for measuring the extracellular acidification rate (ECAR) in vitro, complying with the manufacturer's protocol. Briefly, transfected NSCLC cells $\left(2 \times 10^{4}\right.$ cells/well) were inoculated into an XF96well plate to attach for 16-20 $\mathrm{h}$ under the culture conditions. After that, cell medium was replaced with XF assay unbuffered medium complemented with $2 \mathrm{mM}$ glutamine, followed by the injection of $10 \mathrm{mM}$ glucose, $1 \mu \mathrm{M}$ oligomycin, and $80 \mathrm{mM}$ 2-deoxyglucose. The measured ECAR was recorded as $\mathrm{mpH} / \mathrm{min}$.

\section{Assessment of glucose consumption and lactate production}

Non-small cell lung cancer cells were seeded into 24-well plates overnight. After cell transfection, the glucose consumption and lactate production levels were assayed from cell lysates via the glucose uptake colorimetric assay kit and lactate colorimetric assay kit (BioVision, Milpitas, (A, USA), respectively, according to the operating procedures [24].

\section{Western blot}

The protein was extracted using radio immunoprecipitation assay (RIPA) lysis buffer (Sangon), and the separation of $40 \mu \mathrm{g}$ proteins was performed by sodium dodecyl sulfate polyacrylamide gel electrophoresis (SDS-PAGE), followed by transfer onto polyvinylidene fluoride membranes (Millipore, Billerica, MA, USA) and blockage by $5 \%$ non-fat milk (Sigma, St. Louis, MO, USA). The membranes were incubated with primary antibodies: anti-glucose transporter 1 (anti-GLUT1; Abcam, Cambridge, UK, ab115730, 1 : 1000), anti-lactic dehydrogenase (antiLDHA; Abcam, ab101562, 1 : 1000), anti-TMEM14A (Sigma, HPA072536, 1 : 1000), internal control anti-GAPDH (Abcam, ab181602, 1 : 3000) for $4 \mathrm{~h}$ at indoor temperature. Following the incubation of secondary antibody (Abcam, ab205718, 1 : 5000) for $1 \mathrm{~h}$, the presence of immunoconjugated signals was conducted by the enhanced chemiluminescence reagent (Millipore), then Image Lab software version 4.1 (Bio-Rad Laboratories, Hercules, CA, USA) was used for collecting images and analyzing signal density [25].

\section{Dual-luciferase reporter assay}

The amplified circ_0001421 sequences, including wild-type (WT, containing the putative miR409-3p sites) and mutant-type (MUT, containing the mutant miR-409-3p sites), were constructed into psiCHECK-2 luciferase basic vector (Promega, Madison, WI, USA) to obtain the recombinant luciferase reporters circ_0001421 WT and circ_0001421 MUT. The 3'-UTRs of TMEM14A WT and MUT were also fused to acquire TMEM14A 3'-UTR WT and TMEM14A 3'-UTR MUT. NSCLC cells were transfected with the constructs and miR-409$3 p$ or miR-NC for $48 \mathrm{~h}$, and cell lysate by the passive buffer (Promega) was used for examination of the luciferase intensity by the dual-luciferase reporter system (Promega), abiding by the producer's manual. The relative luciferase activity was expressed as the ratio of firefly/Renilla luciferase intensity.

\section{Xenotransplantation assay}

The A549 cells stably expressing sh-circ_0001421 were constructed through the transfection of lenti- 
virus vector containing short hairpin RNA (shRNA) targeting circ_0001421 (sh-circ_0001421; GenePharma, Shanghai, China), using sh-NC as the negative control. Vital River Laboratory Animal Technology (Beijing, China) provided the purchase service of male BALB/c nude mice ( $n=10$, six-week-old) for us. Then mice underwent subcutaneous injection with transfected A549 cells $\left(1 \times 10^{6} / 50 \mu \mathrm{l} \mathrm{PBS}\right)$, and were divided into sh-circ_0001421 and sh-NC groups (5 mice per group). Tumor burden was monitored through digital calipers weekly, and the acknowledged formula (length $\times$ width $^{2} \times 0.5$ ) was applied for estimating tumor volume. All mice were subjected to euthanasia 5 weeks after injection, then tumors were collected and the weight was measured using an electronic scale. Ultimately, the levels of circ_0001421, miR-409-3p and TMEM14A in the tumors were detected. The animal assay received permission from the Animal Ethics Committee of Xiantao First People's Hospital Affiliated to Changjiang University.

\section{Statistical analysis}

Independently, the experiments in our study were repeated three times. Data were expressed as the mean \pm standard deviation (SD). Statistical analyses were performed using SPSS 20.0 and graphic plotting was conducted through GraphPad

A

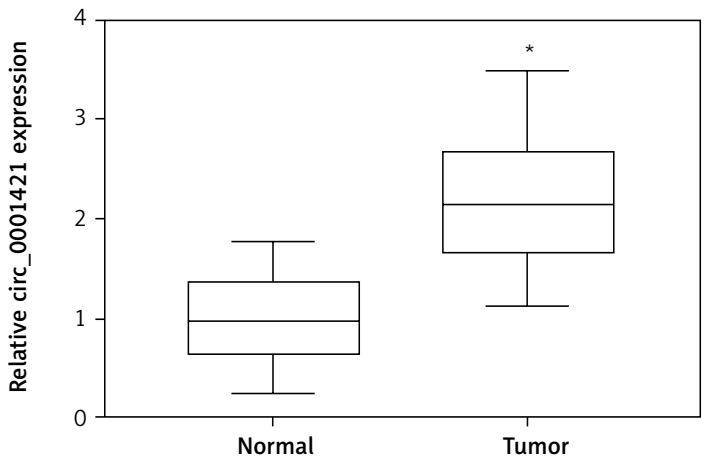

C

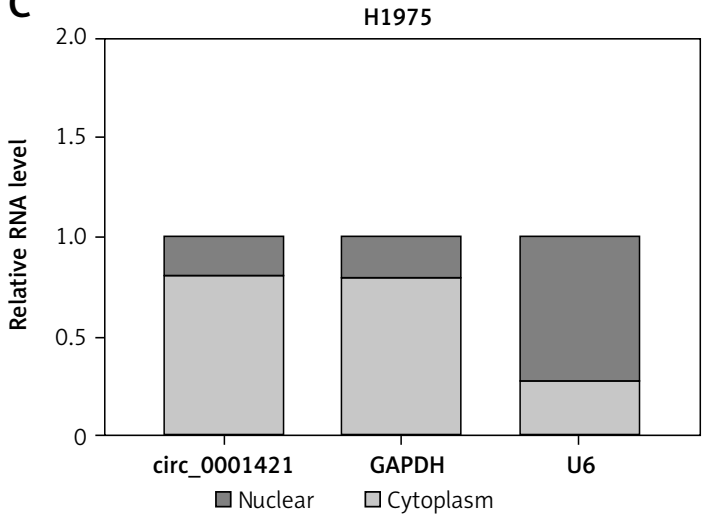

Prism 7. Student's t-test and one-way analysis of variance (ANOVA) followed by Tukey's test were implemented for the difference analysis. A value of $p<0.05$ was considered statistically significant.

\section{Results}

\section{Circ_0001421 was aberrantly overexpressed in NSCLC tissues and cells}

qRT-PCR analysis was used to affirm whether circ_0001421 was dysregulated in NSCLC. As Figure 1 A illustrates, the circ_0001421 level was much higher in NSCLC tissues than that in normal tissues. Also, the overexpression of circ_0001421 was validated in $\mathrm{H} 1975$ and $\mathrm{A} 549$ cells by contrast to normal BEAS-2B cells (Figure $1 \mathrm{~B}$ ). And interestingly, both circ_0001421 and GAPDH were abundant in cytoplasm but small nuclear RNA U6 existed in the nucleus principally, suggesting the location of circ_0001421 in cytoplasm (Figure 1 C, D). Mainly, we verified that circ_0001421 was overexpressed in NSCLC.

\section{Knockdown of circ_0001421 restrained colony formation, migration, invasion and glycolysis of NSCLC cells}

si-circ_0001421 transfection was applied to knock down circ_0001421 for researching the role

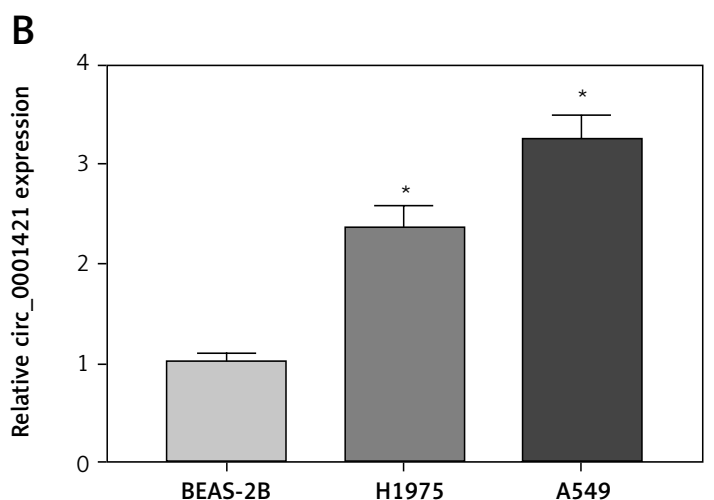

D

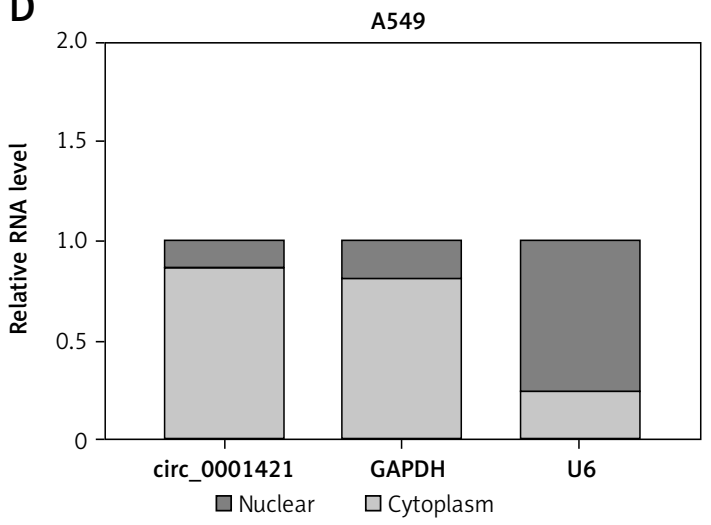

Figure 1. Circ_0001421 was aberrantly overexpressed in NSCLC tissues and cells. A, B - Circ_0001421 level in NSCLC tissues (A) and cells (B) was determined using qRT-PCR. C, D - The analysis of circ_0001421 location was performed by $q$ RT-PCR. ${ }^{*} P<0.05$ 
A

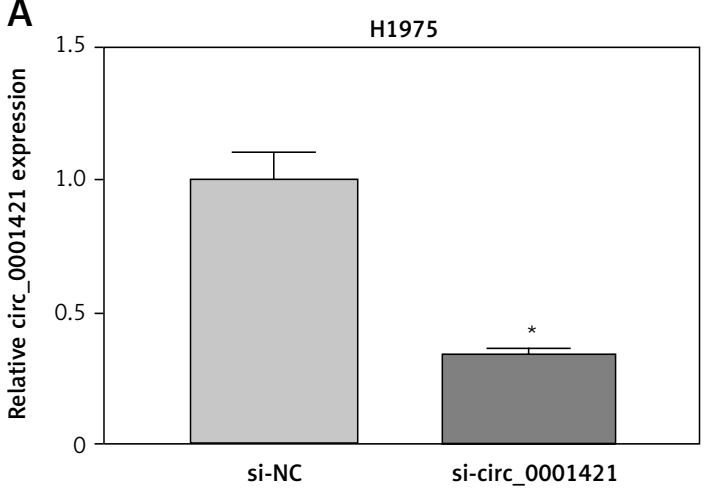

C
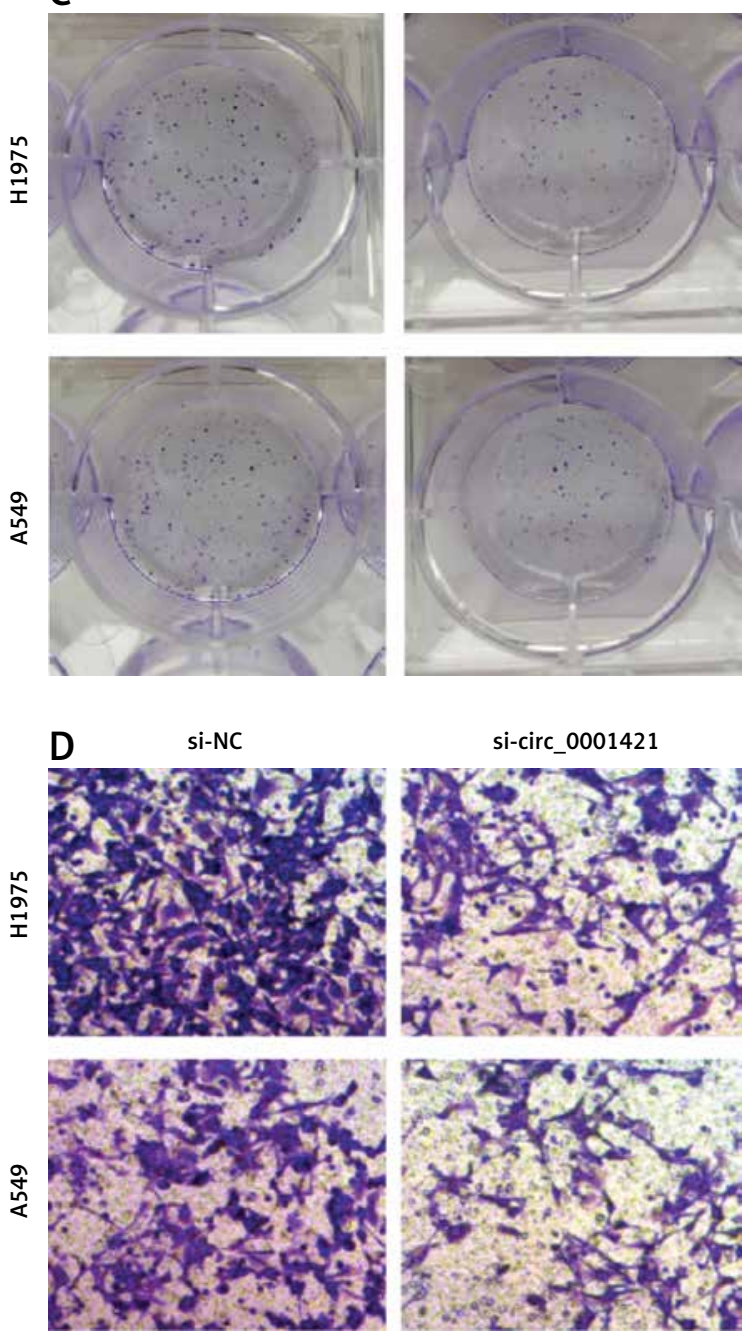

Figure 2. Knockdown of circ 0001421 restrained colony formation, migration, invasion and glycolysis of NSCLC cells. H1975 and A549 cells were transfected with si-circ_0001421 or si-NC. A, B - The knockdown efficiency of si-circ 0001421 in H1975 and A549 cells was analyzed by qRT-PCR. C - The colony formation ability was detected using colony formation assay. D, E - Transwell assay was used for assessing cell migration and invasion

of circ 0001421 in NSCLC cells. QRT-PCR revealed the successful interference of si-circ_0001421 with circ_0001421 expression compared to the si-NC group (Figure $2 \mathrm{~A}$, B). Subsequently, the further function exploration was implemented. In colony formation assay, fewer colonies were observed in the si-circ_0001421 group than the
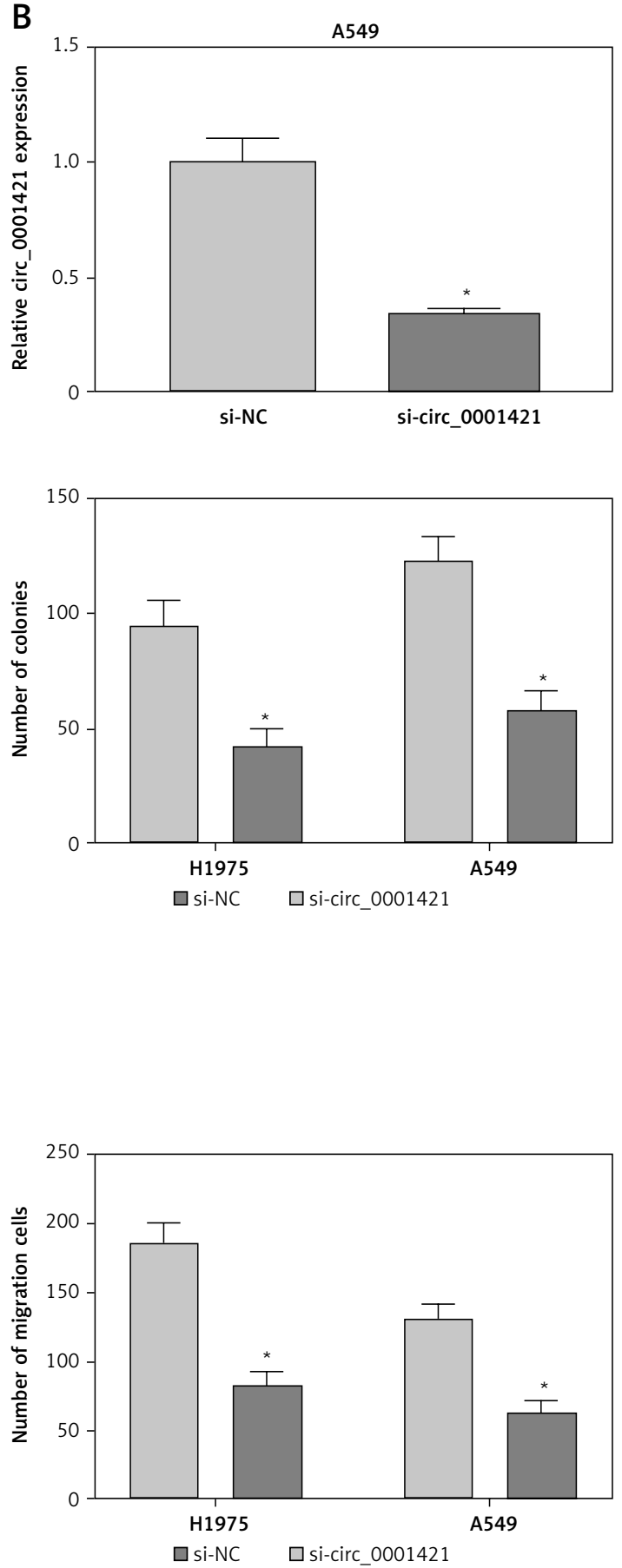

si-NC group (Figure 2 C). Transwell assay showed that the number of migrated (Figure $2 \mathrm{D}$ ) and invaded (Figure $2 \mathrm{E}$ ) cells remarkably declined following the down-regulation of circ_0001421. The indicators of glycolysis are numerous, such as ECAR, glucose consumption, lactate production and the glycolysis-related proteins including glu- 

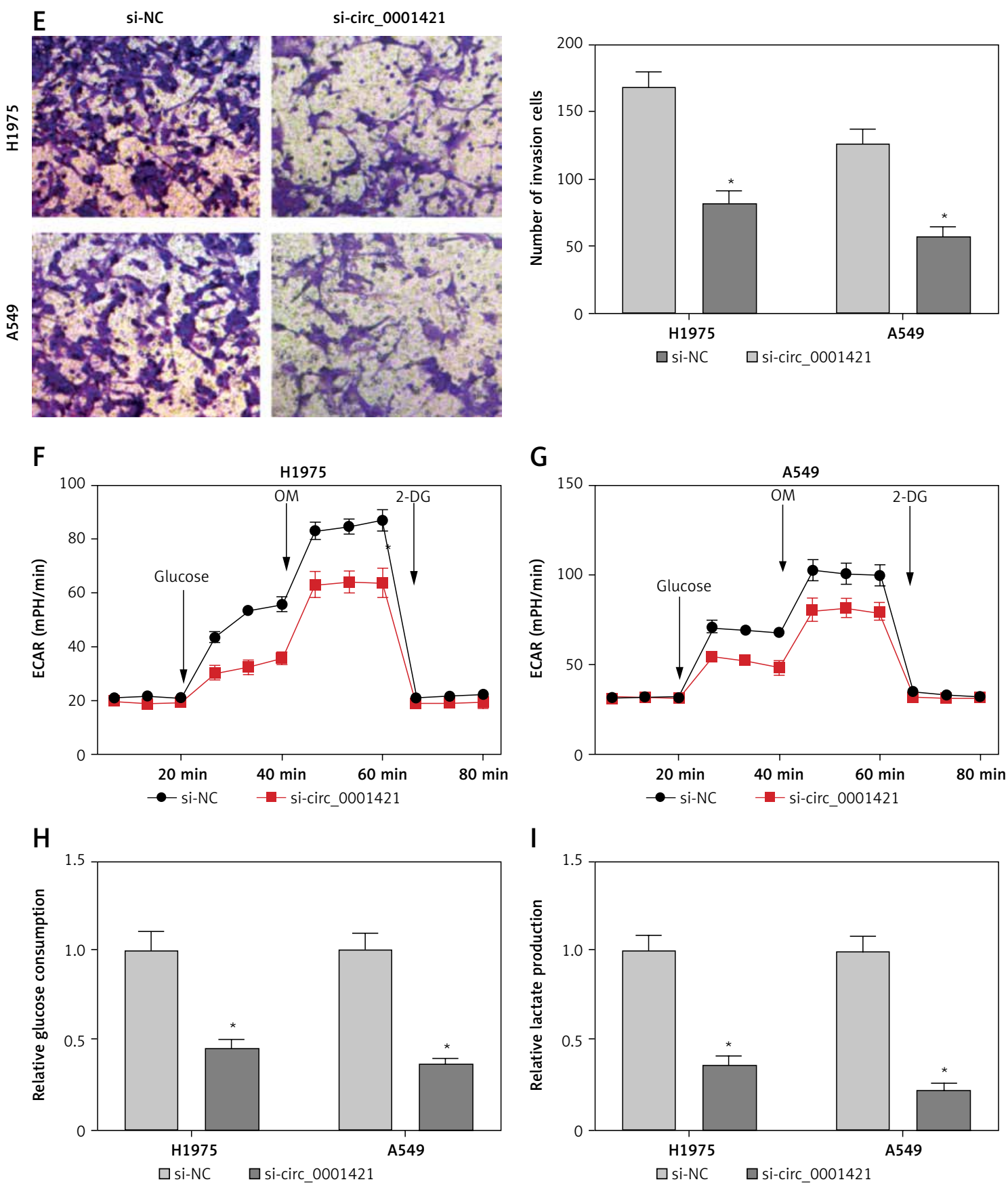

Figure 2. Cont. D, E - Transwell assay was used for assessing cell migration and invasion. F-K - The glycolysis was estimated through ECAR measurement $(\mathrm{F}, \mathrm{G})$, glucose consumption $(\mathrm{H})$, lactate production $(\mathrm{I})$

cose transporter-1 (GLUT1) and lactate dehydrogenase A (LDHA) [26, 27]. The experimental results indicated that circ 0001421 knockdown decreased the ECAR in both H1975 (Figure 2 F) and A549 (Figure $2 \mathrm{G}$ ) cells, as well as the glucose consumption (Figure $2 \mathrm{H}$ ) and lactate production (Figure $2 \mathrm{I}$ ). Western blot revealed that the protein expression levels of GLUT1 and LDHA were repressed in $\mathrm{H} 1975$ (Figure $2 \mathrm{~J}$ ) and $\mathrm{A} 549$ (Figure $2 \mathrm{~K}$ ) cells transfected with si-circ_0001421. Altogether, circ_0001421 down-regulation exerted inhibitory effects on colony formation, migration, invasion and glycolysis in NSCLC cells.

\section{Circ_0001421 served as a sponge of miR-409-3p}

Increasing studies have testified that circRNAs could act as miRNA "sponges" in various human cancers [28-30]. After the prediction of StarBase v2.0 software, we noted that circ_0001421 contained the binding sites of miR-409-3p (Figure $3 \mathrm{~A}$ ). 
H1975

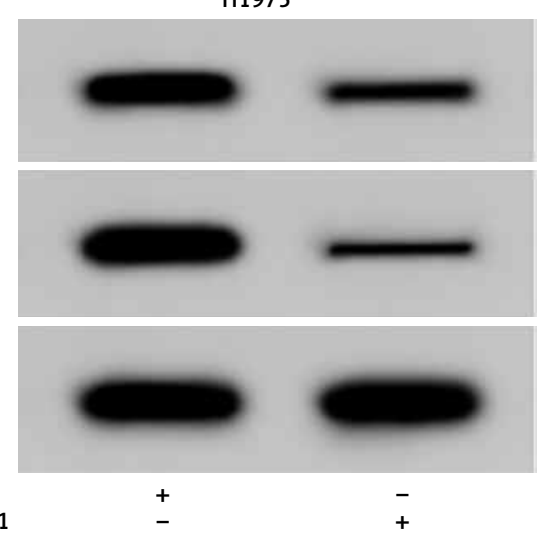

si-NC si-circ_000142

K

GLUT1

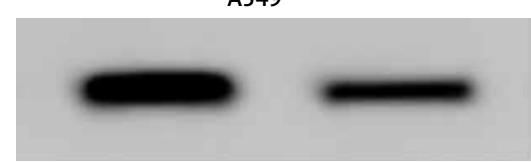

LDHA

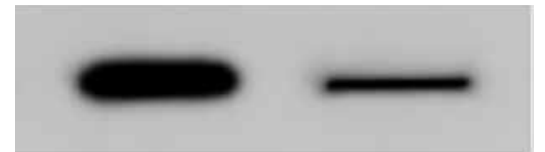

GAPDH

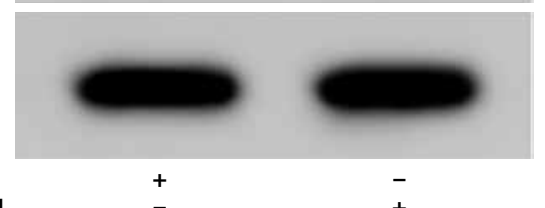

si-circ_000142
H1975

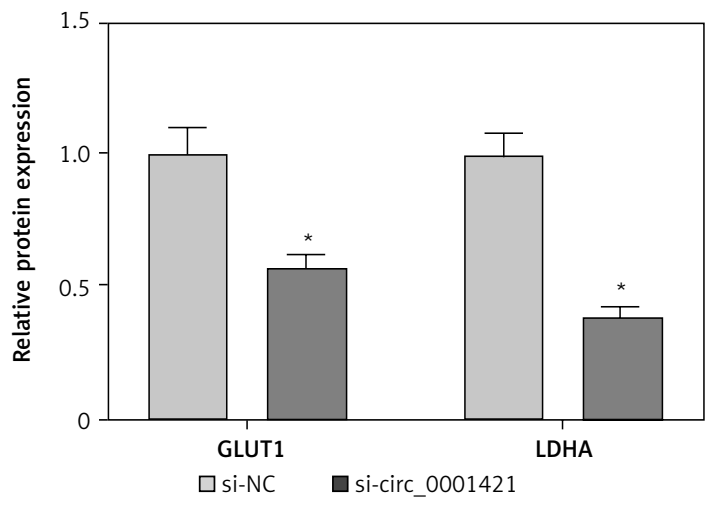

A549

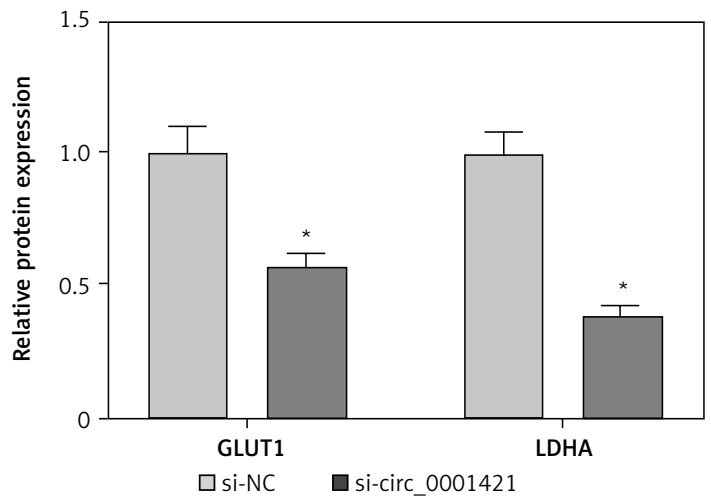

Figure 2. Cont. The protein levels of GLUT1 and LDHA by Western blot (J, K). ${ }^{\star} P<0.05$

The interaction between circ_0001421 and miR409-3p was certified by dual-luciferase reporter assay. As shown in Figure 3 B, C, miR-409-3p co-transfection with circ_0001421 WT strikingly reduced the relative luciferase activity of $\mathrm{H} 1975$ and A549 cells, but there was no conspicuous difference in luciferase activity after miR-409-3p co-transfection with circ_0001421 MUT. In comparison to normal tissues and BEAS-2B cells, miR409-3p expression was significantly reduced in both NSCLC tissues (Figure 3 D) and cells (Figure $3 \mathrm{E}$ ). And notably, miR-409-3p level was up-regulated in H1975 (Figure $3 \mathrm{~F}$ ) and A549 (Figure $3 \mathrm{G}$ ) cells transfected with si-circ_0001421, which implied that circ_0001421 acted as a miR-409-3p sponge.

\section{Inhibition of miR-409-3p reverted the impacts of circ_0001421 knockdown on NSCLC cells}

Next, we used rescue experiments to study the regulatory relation between circ-0001421 and miR-409-3p. qRT-PCR demonstrated that antimiR-409-3p transfection significantly recovered the si-circ_0001421-induced increase of miR-409$3 p$ expression, suggesting that the inhibitory effect of anti-miR-409-3p on miR-409-3p level was excellent (Figure $4 \mathrm{~A}, \mathrm{~B}$ ). In addition, the repression of colony formation (Figure $4 \mathrm{C}, \mathrm{D}$ ), migration (Figure 4 E, F) and invasion (Figure 4 G, H) caused by circ_0001421 depression in H1975 and A549 cells were all prominently reversed following the inhibition of miR-409-3p level. Seahorse analysis showed that the si-circ_0001421-motivated suppressive effect on ECAR was reversed by miR-409$3 p$ inhibitor (Figure 4 I, J). Also, si-circ_0001421 and anti-miR-409-3p co-transfection enhanced the glucose consumption (Figure $4 \mathrm{~K}, \mathrm{~L}$ ) and lactate production (Figure $4 \mathrm{M}, \mathrm{N}$ ) contrasted to the sicirc_0001421 + anti-miR-NC group. And the introduction of anti-miR-409-3p offset the down-regulation of GLUT1 and LDHA protein expression evoked by the low expression of circ_0001421 in H1975 (Figure 4 O) and A549 (Figure 4 P) cells. All the data proved that the impacts of circ_0001421 knockdown on NSCLC cells were relieved through inhibiting miR-409-3p.

\section{TMEM14A was a target of miR-409-3p and circ_0001421 regulated TMEM14A expression by sponging miR-409-3p}

MiRNAs can usually interact with the 3'UTR of downstream gene [31, 32]. StarBase v2.0 displayed 
A $\begin{array}{ll}\text { circ_0001421 WT } & \text { 5' cucagcaauugGAUGCAACAUUu 3' } \\ \text { miR-409-3p } & \text { 3' uccccaaguggCU--CGUUGUAAg 5' } \\ \text { circ_0001421 MUT } & \text { 5' cucagcaauugAGUAUCGACCGu 3' }\end{array}$

B

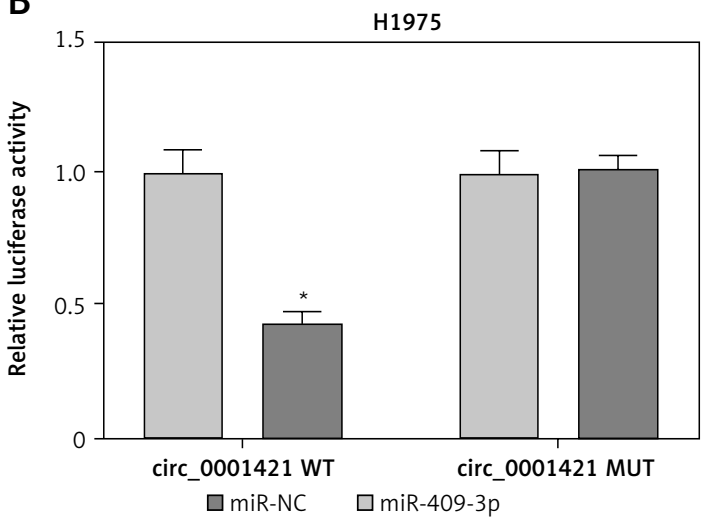

D

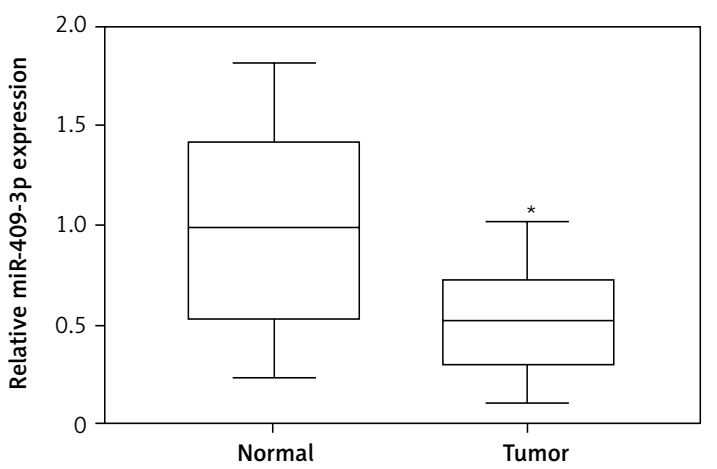

$\mathbf{F}$

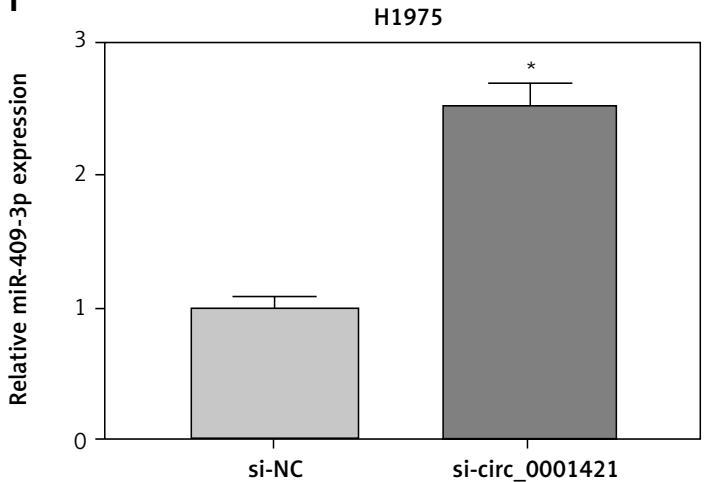

C

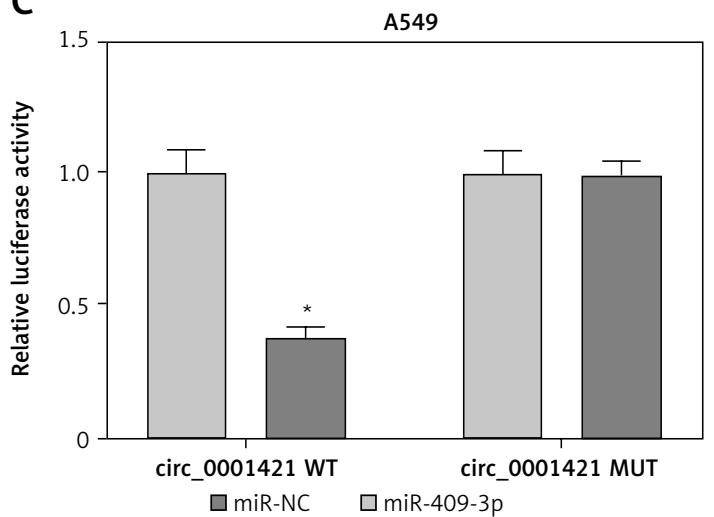

$\mathrm{E}$

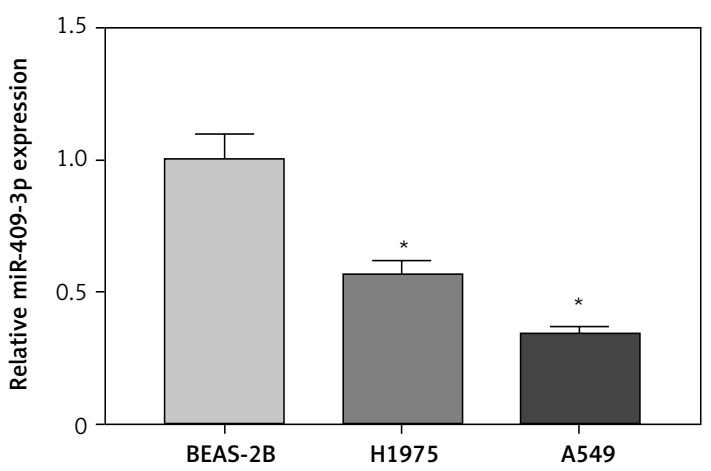

G

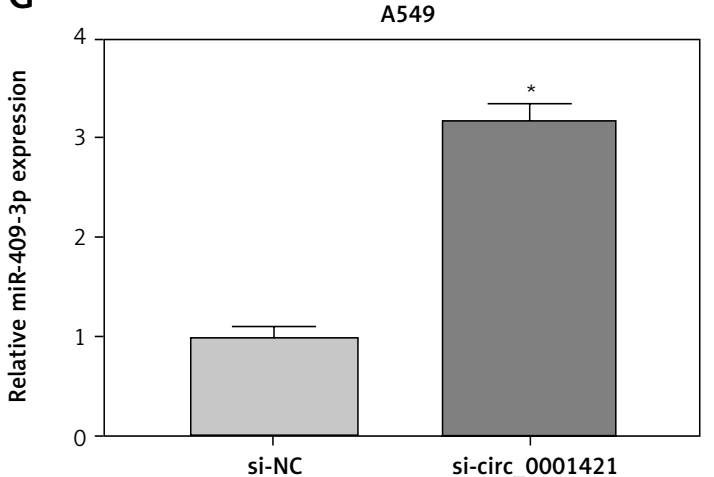

Figure 3. Circ_0001421 served as a sponge of miR-409-3p. A - The bioinformatic analysis between circ_0001421 and miR-409-3p was performed by StarBase v2.0. B, C - The correlation between circ 0001421 and miR-409-3p was analyzed using the dual-luciferase reporter assay. D, E-The level of miR-409-3p in NSCLC tissues and cells was detected through qRT-PCR. F, G - The qRT-PCR was applied for assessing the influence of circ 0001421 inhibition on the expression of miR-409-3p in NSCLC cells. ${ }^{*} P<0.05$

the binding sites between TMEM14A 3'UTR and miR-409-3p (Figure $5 \mathrm{~A}$ ). Furthermore, the luciferase activity of the TMEM14A 3'UTR WT group was refrained by miR-409-3p overexpression, while it remained unchanged in the TMEM14A 3'UTR MUT group (Figure $5 \mathrm{~B}, \mathrm{C}$ ). After verifying the combination of miR-409-3p and TMEM14A, we measured the expression of TMEM14A in NSCLC. Distinctly,
NSCLC tissues (Figure $5 \mathrm{D}$ ) and cells (Figure $5 \mathrm{E}$ ) expressed a rising tendency of TMEM14A protein level, compared to normal tissues and cells. After miR409-3p was transfected into H1975 (Figure 5 F) and A549 (Figure $5 \mathrm{G}$ ) cells, the decrease of TMEM14A protein expression was presented by Western blot. Moreover, transfection of si-circ_0001421 triggered the down-regulation of TMEM14A, which 
A

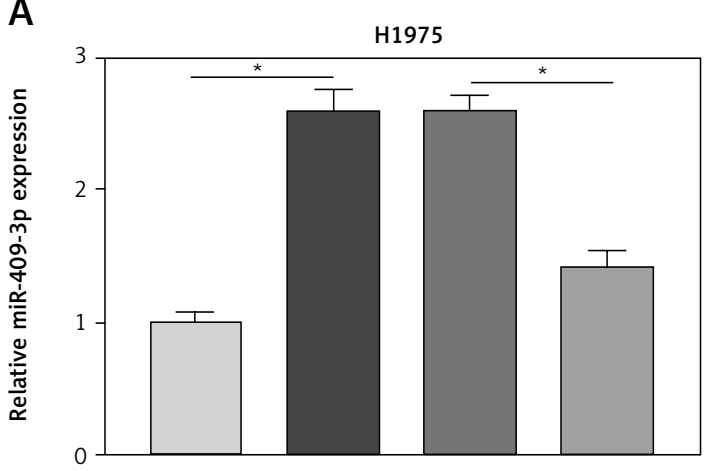

C

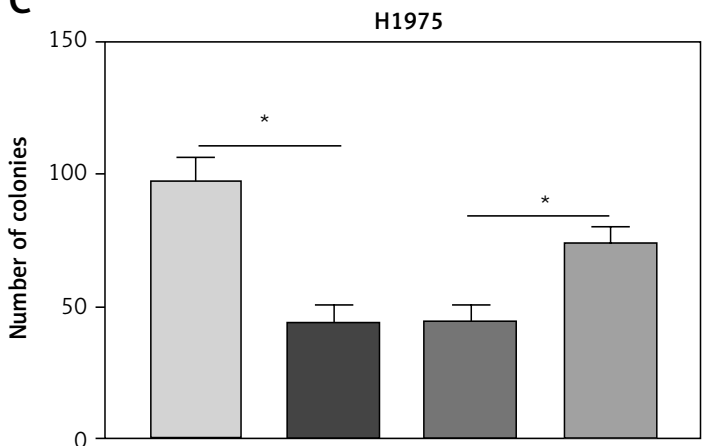

E

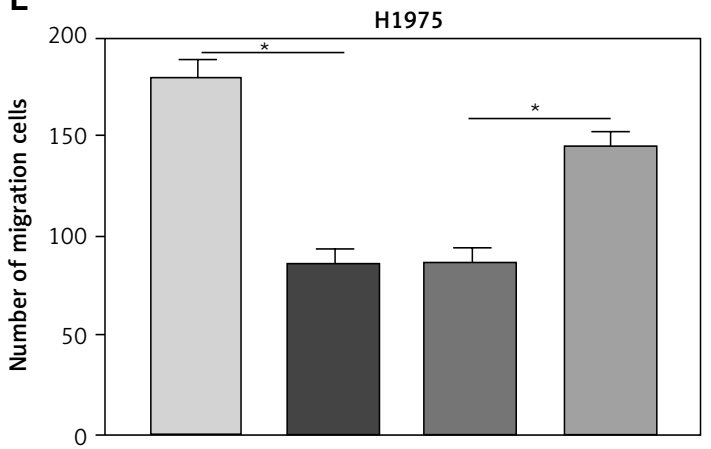

G

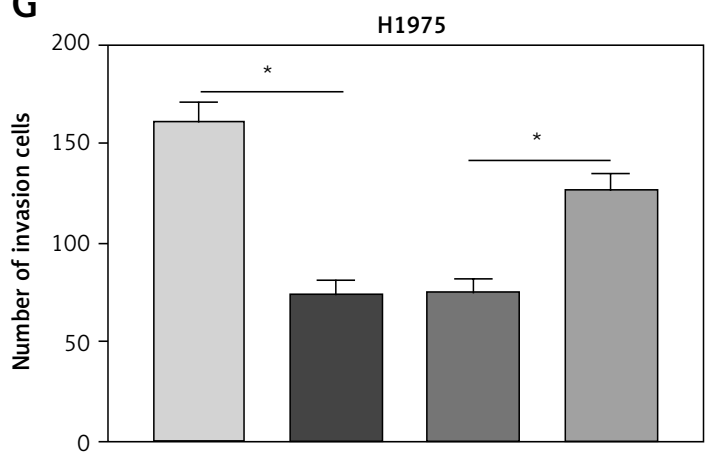

B

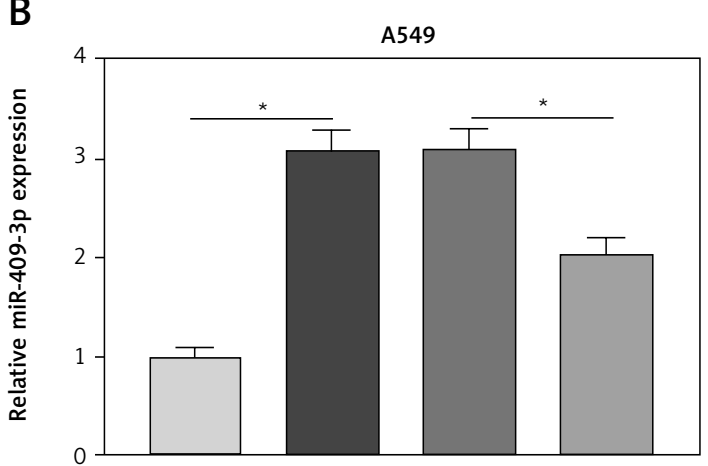

D

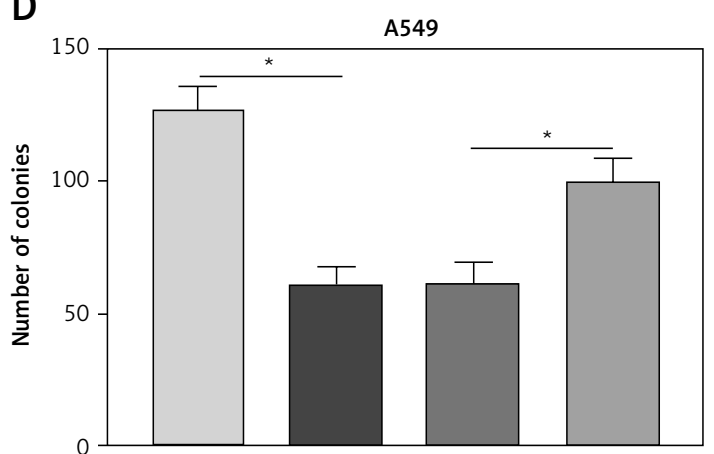

F

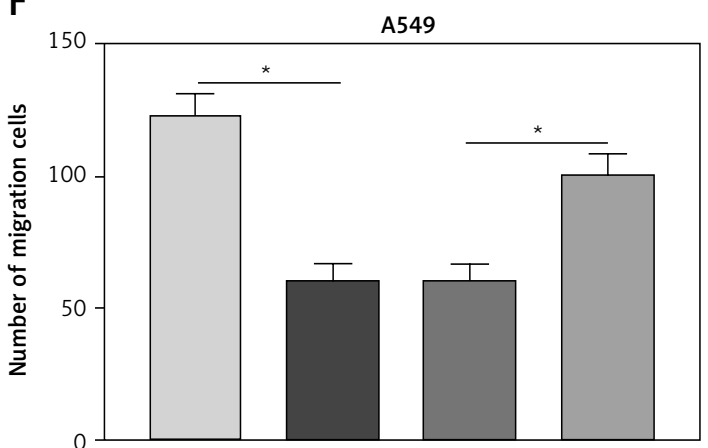

$\mathrm{H}$

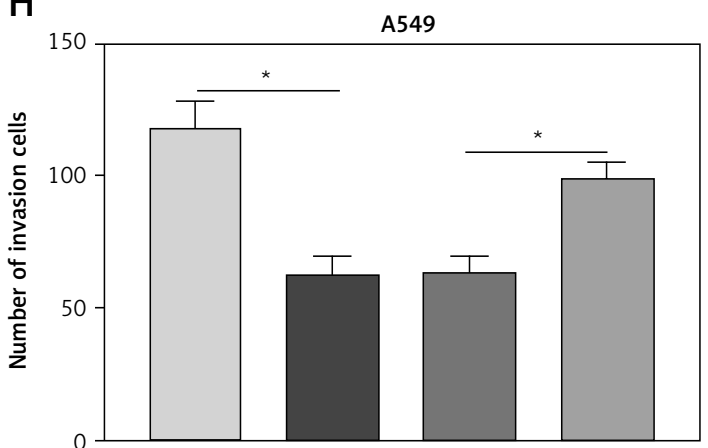

$\square$ si-NC

口 si-circ_0001421

$\square$ si-circ $0001421+$ anti-miR-NC

$\square$ si-circ $0001421+$ anti-miR-409-3p

Figure 4. Inhibition of miR-409-3p reversed the impacts of circ 0001421 knockdown on NSCLC cells. H1975 and A549 cells were transfected with si-NC, si-circ_0001421, si-circ_0001421 + anti-miR-NC or si-circ_0001421 + antimiR-409-3p. A, B - miR-409-3p expression was determined using qRT-PCR. C, D - Colony formation assay was performed to evaluate the colony ability of cells. $\mathbf{E}-\mathbf{H}-$ Cell migration $(\mathbf{E}, \mathbf{F})$. Invasion $(\mathbf{G}, \mathbf{H})$ were analyzed by transwell assay. 

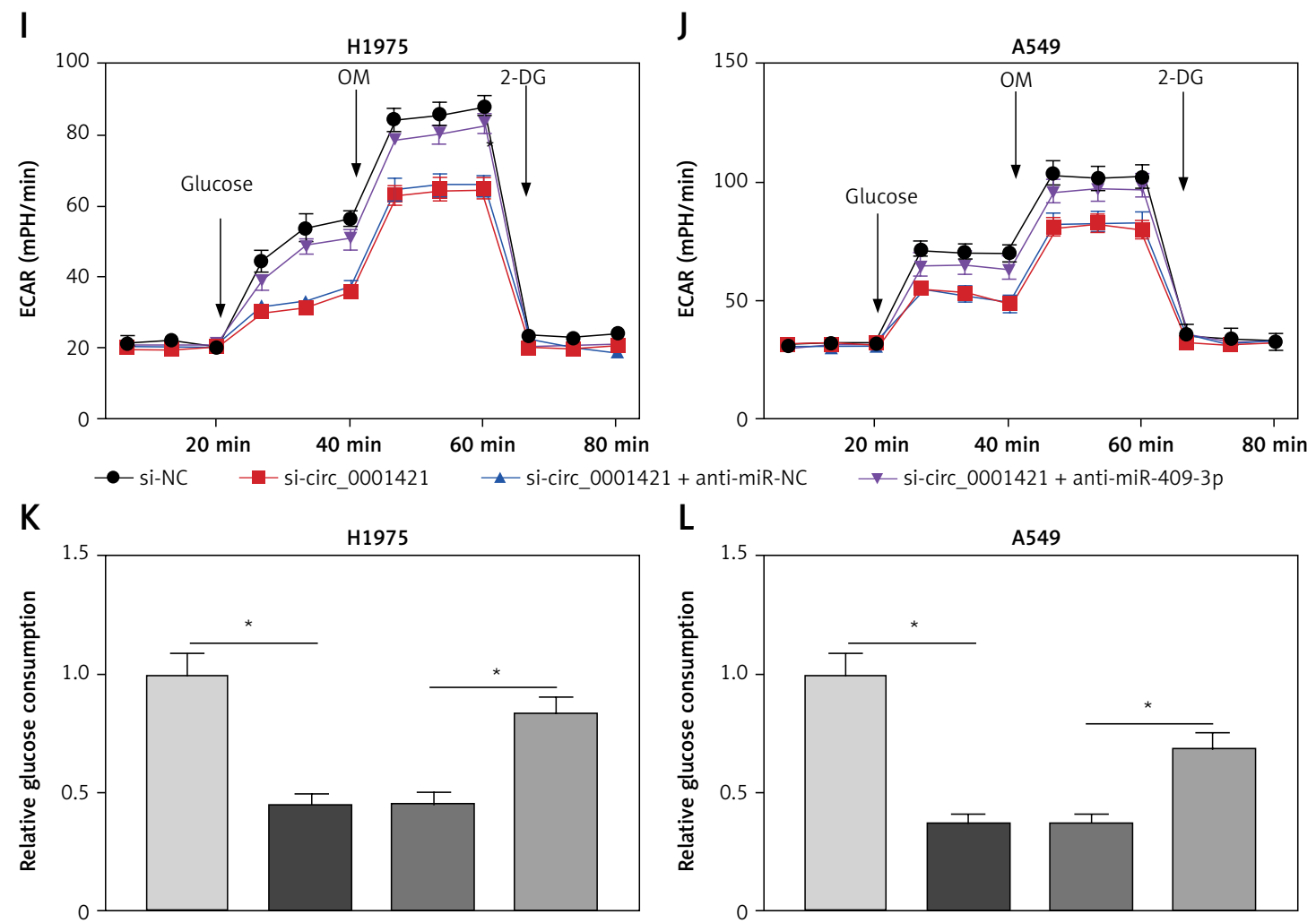

$\mathrm{L}$
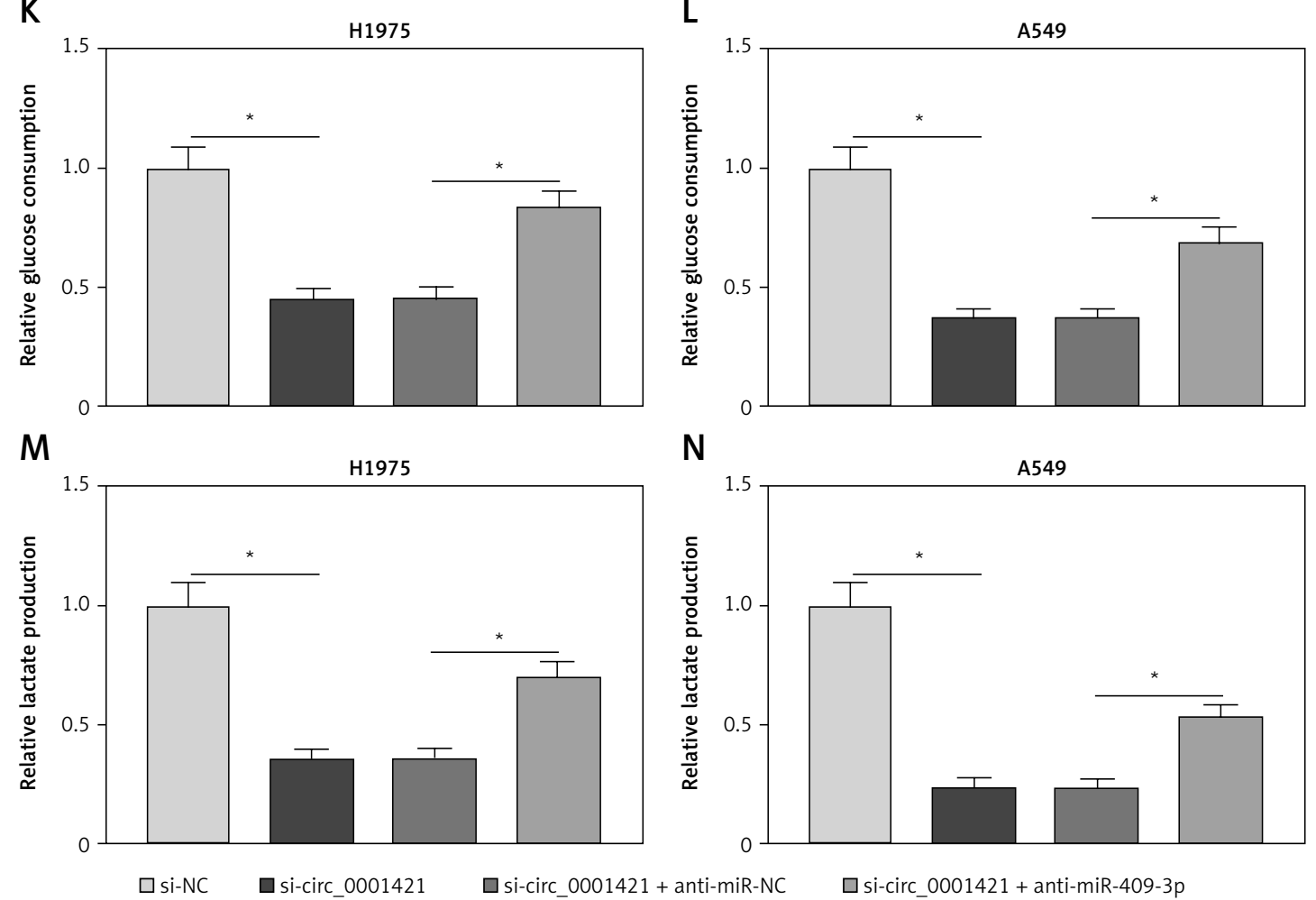

0 H1975
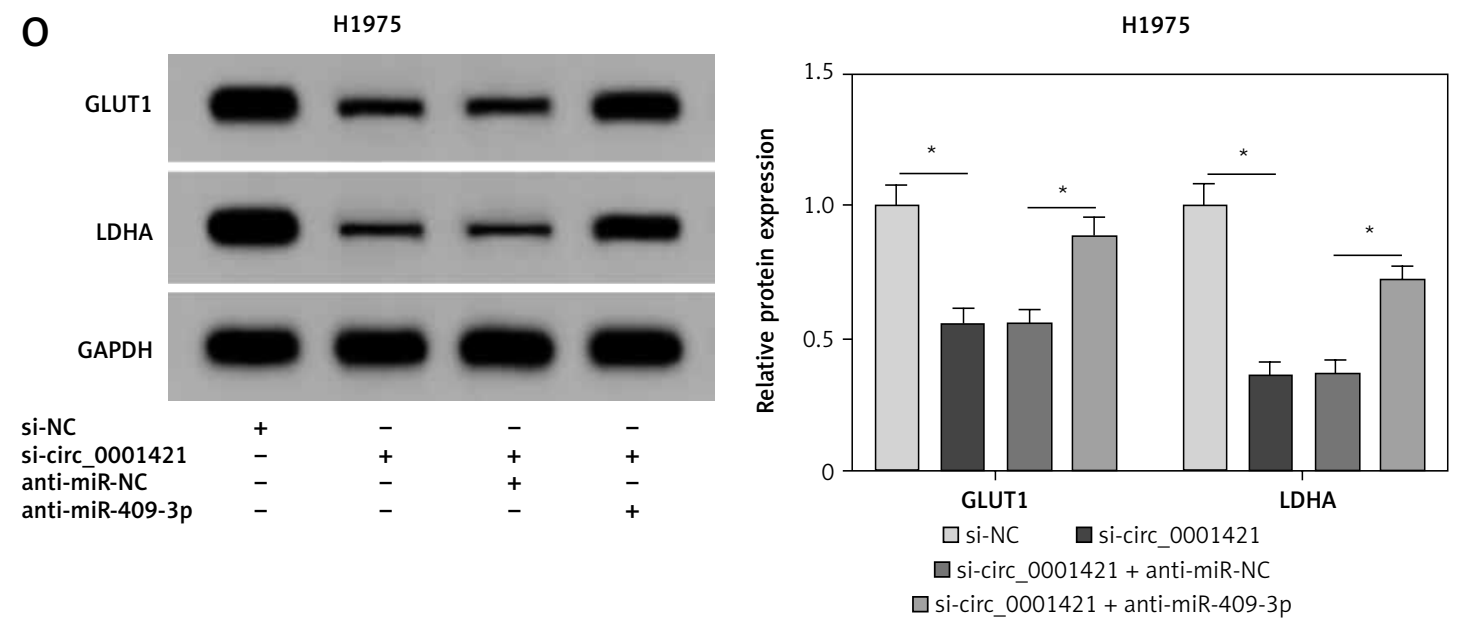

Figure 4. Cont. I-P - The ECAR detection (I, J), glucose consumption (K, L). Lactate production ( $\mathbf{M}, \mathbf{N})$ and the GLUT1 and LDHA protein expression by Western blot $(\mathbf{O}, \mathbf{P})$ were implemented for assessing the glycolysis metabolism. ${ }^{*} P<0.05$ 
P

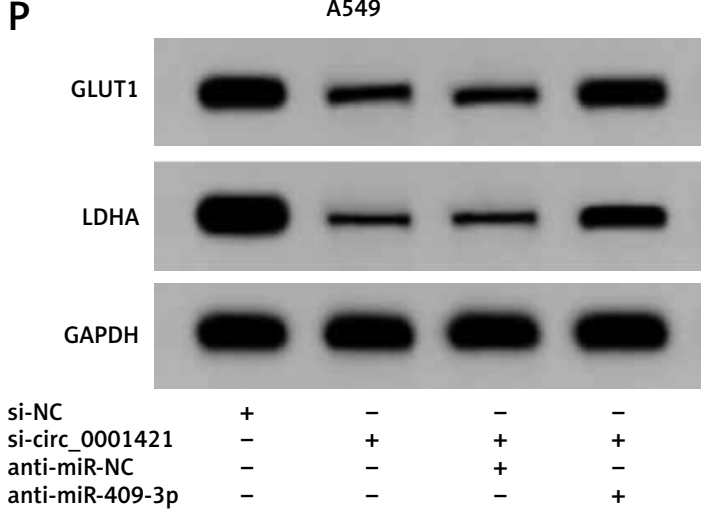

A549

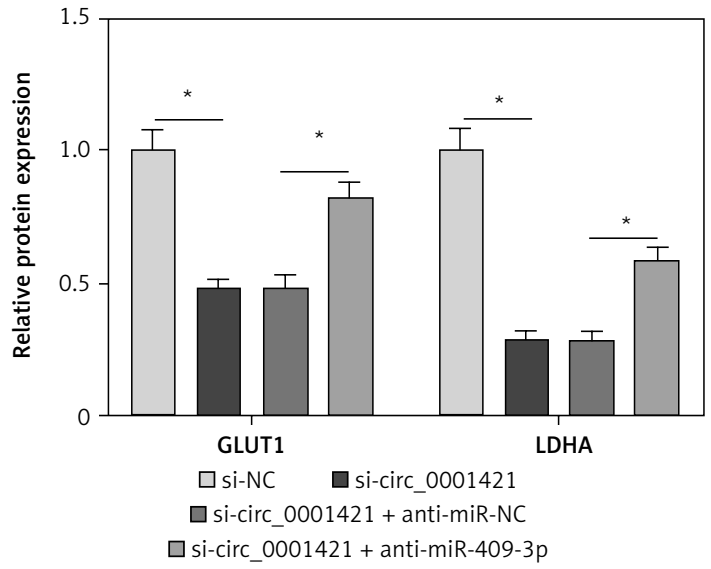

Figure 4. Cont. LDHA protein expression by Western blot $(\mathbf{O}, \mathbf{P})$ were implemented for assessing the glycolysis metabolism. ${ }^{\star} P<0.05$

A

$\begin{array}{ll}\text { TMEM14A 3' UTR WT 5' cucagcaauugGAUGCAACAUUu 3' } & \\ \text { miR-409-3p } & \text { 3' uccccaaguggCU--CGUUGUAAg 5' } \\ \text { TMEM14A 3' UTR MUT 5' cucagcaauugAGUAUCGACCGu 3' }\end{array}$
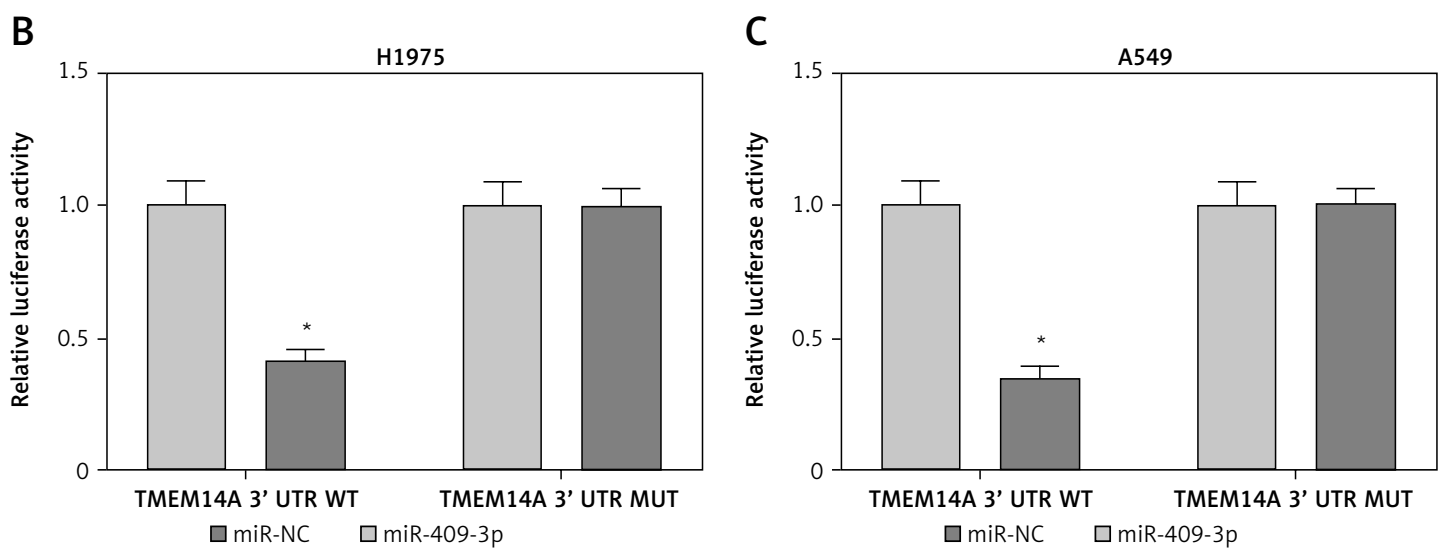

D
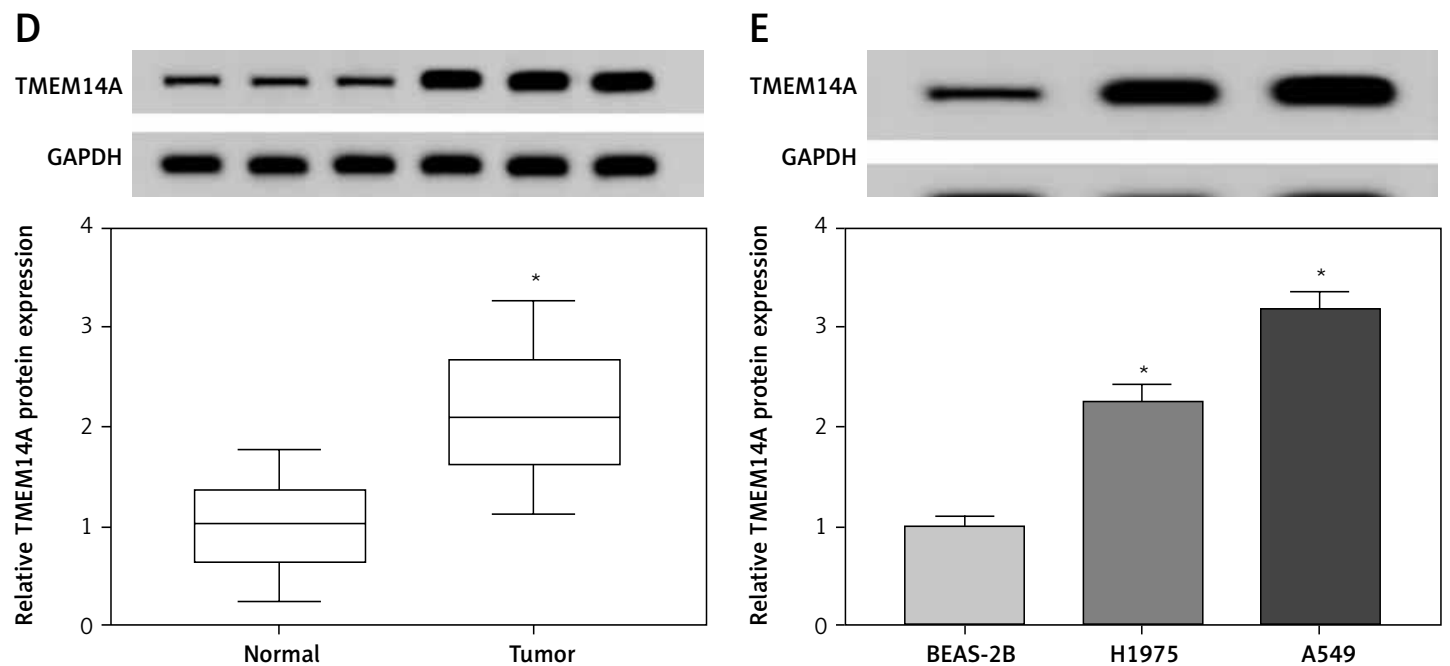

Figure 5. TMEM14A was a target of miR-409-3p and circ_0001421 regulated TMEM14A expression by sponging miR-409-3p. A - The binding sites of miR-409-3p and TMEM14A were analyzed by StarBase v2.0. B, C - Dualluciferase reporter assay was used for confirming the combination between miR-409-3p and TMEM14A. D, E - TMEM14A protein expression in NSCLC tissues and cells was examined by Western blot. 
F
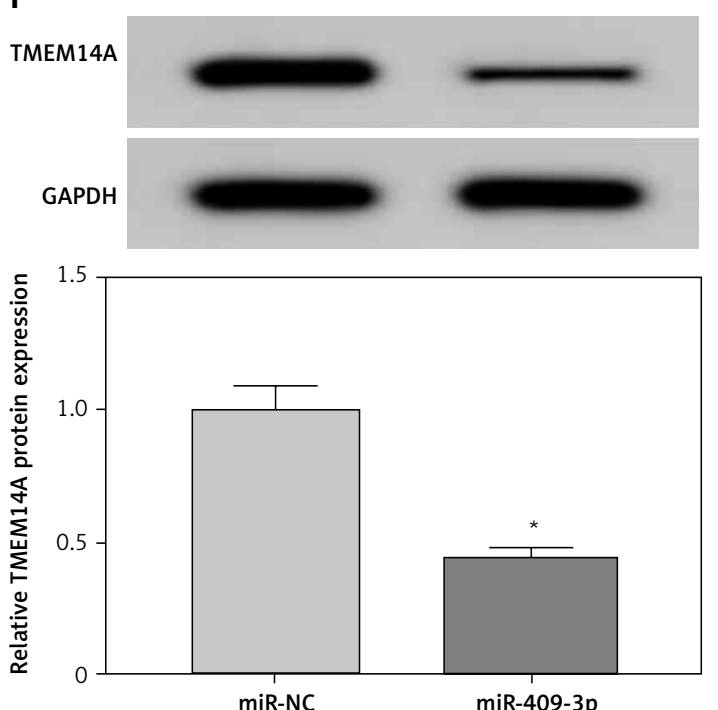

$\mathrm{H}$
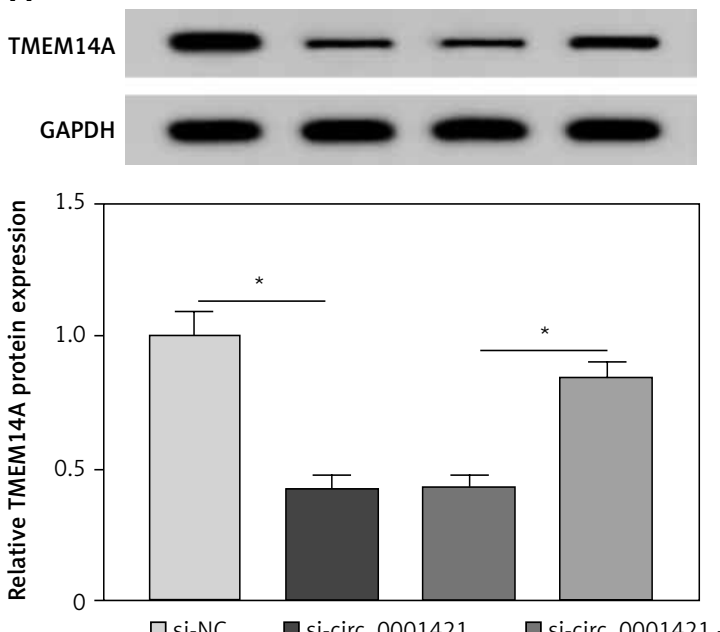

G
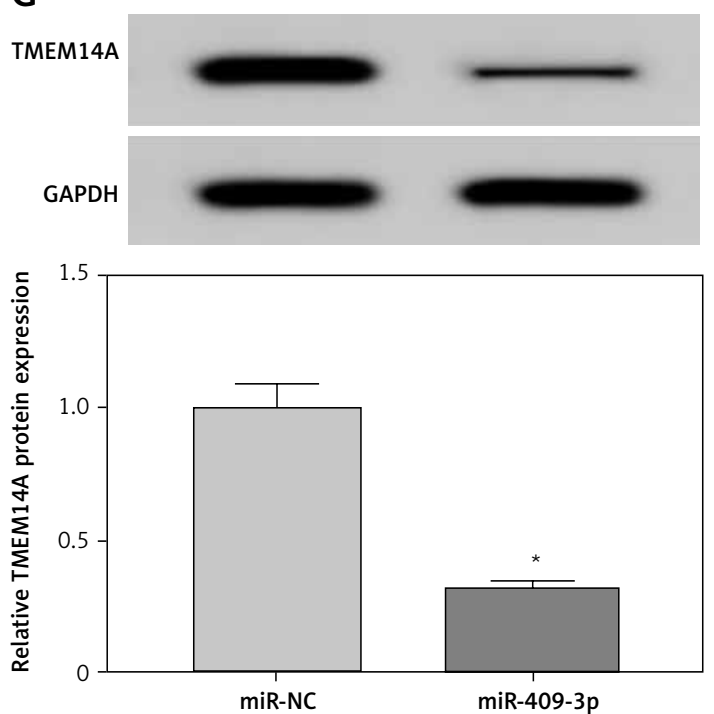

miR-NC

A549
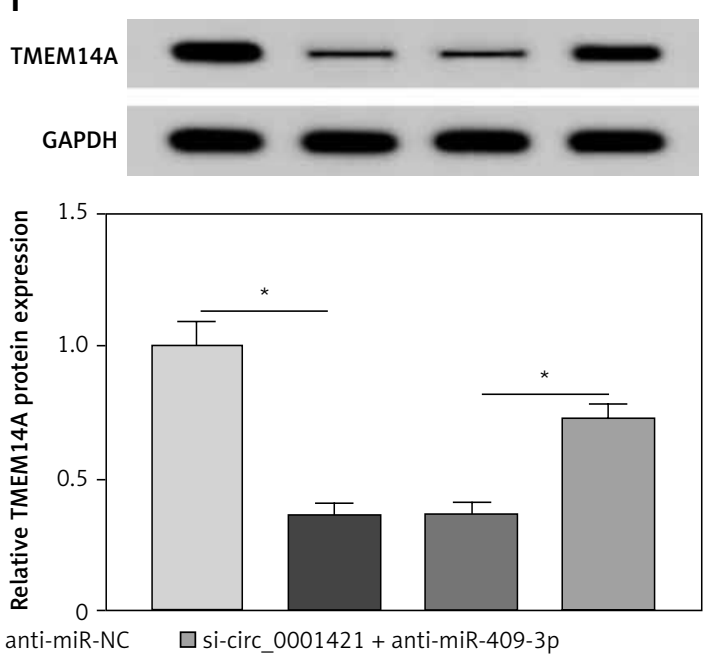

Figure 5. Cont. F, G - Western blot was applied to assess the effect of miR-409-3p on TMEM14A expression with miR-NC as the negative control. H, I - The protein level of TMEM14A was detected in H1975 and A549 cells transfected with si-circ_0001421, si-circ_0001421 + anti-miR-409-3p or matched controls. ${ }^{*} P<0.05$

was partly rescued through miR-409-3p depletion (Figure $5 \mathrm{H}, \mathrm{l}$ ). These results indicated that miR409-3p targeted TMEM14A and circ_0001421 modulated TMEM14A via sponging miR-409-3p.

\section{MiR-409-3p overexpression suppressed} colony formation, migration, invasion and glycolysis via targeting TMEM14A in NSCLC cells

H1975 and A549 cells were transfected with miR-NC, miR-409-3p, miR-409-3p + vector or miR-409-3p + TMEM14A to research whether TMEM14A was responsible for the tumor inhibitor role of miR-409-3p in NSCLC. Firstly, TMEM14A was successfully overexpressed by TMEM14A transfection, which alleviated the miR-409-3p-in- duced restraint of TMEM14A protein expression (Figure $6 \mathrm{~A}, \mathrm{~B}$ ). The overexpression of miR-409$3 p$ resulted in the reduction of the number of cell colonies (Figure $6 \mathrm{C}, \mathrm{D}$ ), migration (Figure $6 \mathrm{E}, \mathrm{F}$ ) and invasion (Figure $6 \mathrm{G}, \mathrm{H}$ ), whereas ectopic high expression of TMEM14A neutralized these effects. Additionally, the miR-409-3p-induced repressive influence on ECAR was abrogated following TMEM14A up-regulation in part (Figure $6 \mathrm{I}$, J). Likewise, miR-409-3p transfection evoked the inhibition of glucose consumption (Figure $6 \mathrm{~K}, \mathrm{~L}$ ) and lactate production (Figure $6 \mathrm{M}, \mathrm{N}$ ) as well as the protein levels of GLUT1 and LDHA (Figure 6 O, P), while this suppression was counteracted by the promotion of TMEM14A. We concluded that the tumor inhibitory function of miR-409-3p in NSCLC was achieved by targeting TMEM14A. 
A
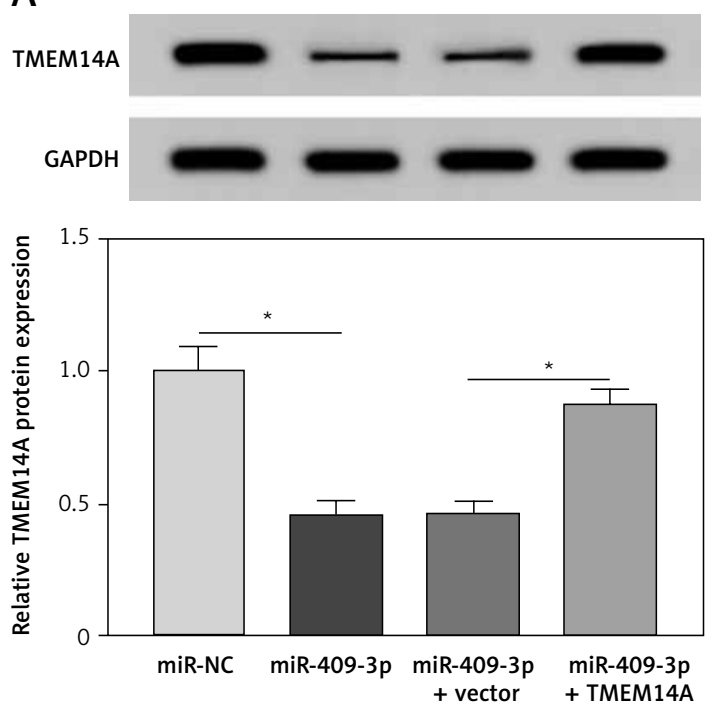

C

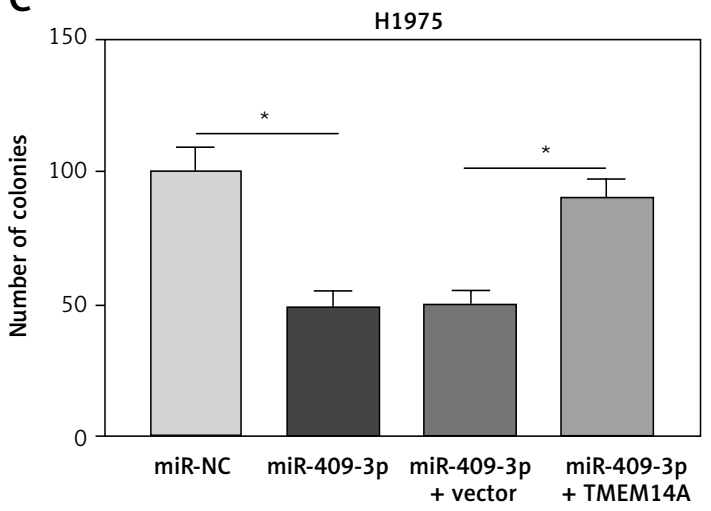

$\mathrm{E}$

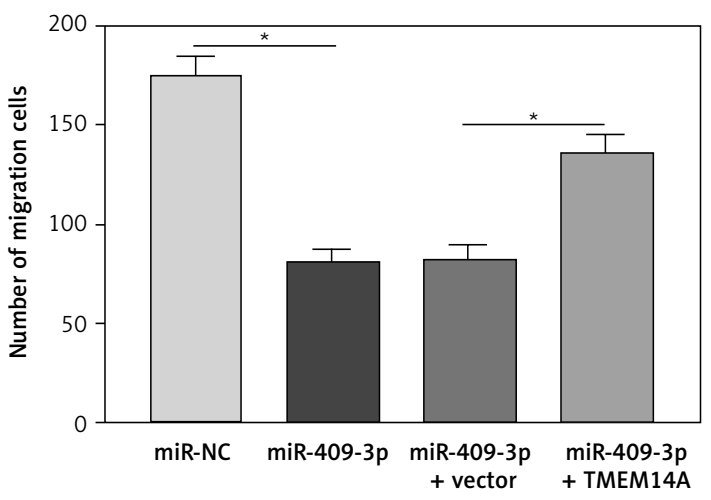

B
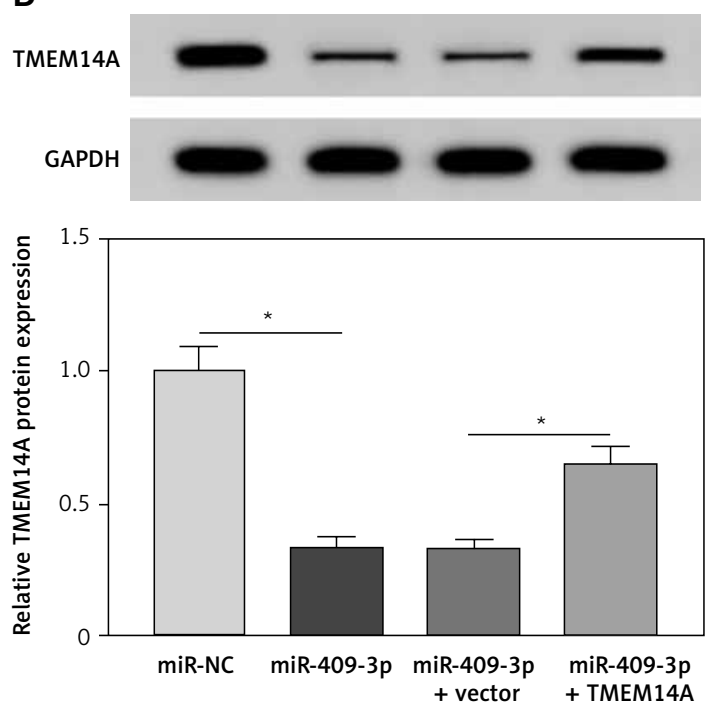

D

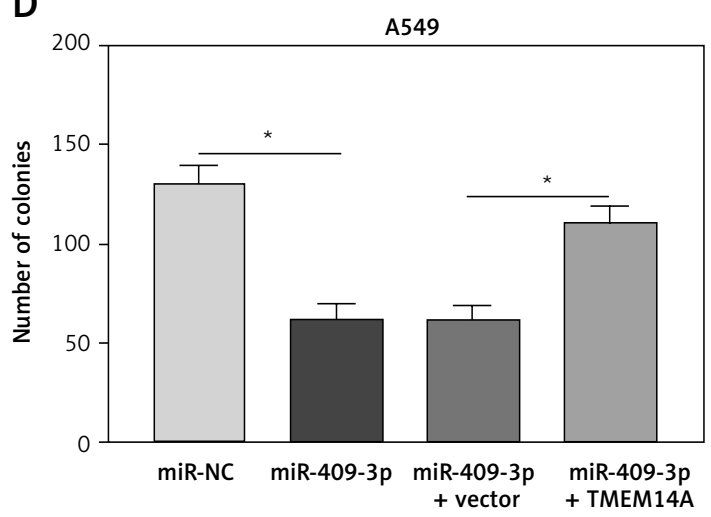

$\mathbf{F}$

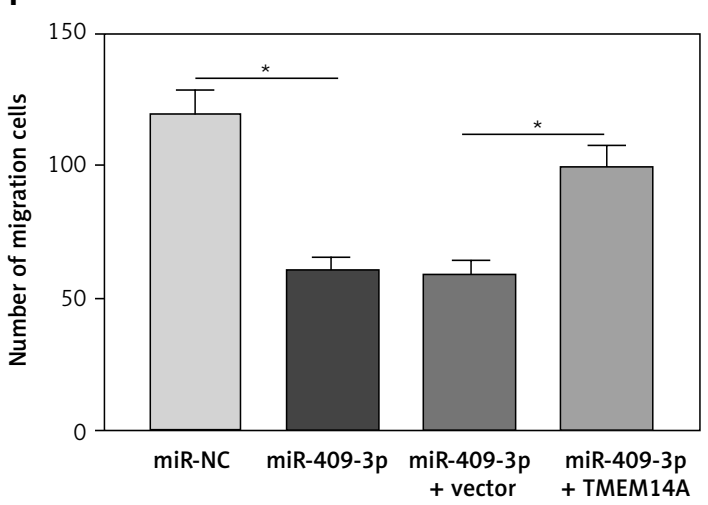

Figure 6. MiR-409-3p overexpression suppressed colony formation, migration, invasion and glycolysis via targeting TMEM14A in NSCLC cells. H1975 and A549 cells were transfected with miR-NC, miR-409-3p, miR-409-3p + vector or miR-409-3p + TMEM14A. A, B - Western blot was conducted for the determination of TMEM14A protein level. C, D - The detection of colony ability was performed using the colony formation assay. $\mathbf{E}-\mathbf{H}-$ The evaluation of cell migration $(\mathbf{E}, \mathbf{F})$ and invasion $(\mathbf{G}, \mathbf{H})$ was administered through the transwell assay

Depression of circ_0001421 reduced tumor growth of NSCLC via regulation of the miR409-3p/TMEM14A axis in vivo

The effect of circ_0001421 on NSCLC in vivo was investigated by xenotransplantation assay.
We observed the evident decrease of tumor volume (Figure $7 \mathrm{~A}$ ) and weight (Figure $7 \mathrm{~B}$ ) in the sh-circ_0001421 group, by comparison with the sh-NC group. Also, knockdown of circ_0001421 clearly reduced the expression of circ_0001421 (Figure 7 C) but enhanced the miR-409-3p level 

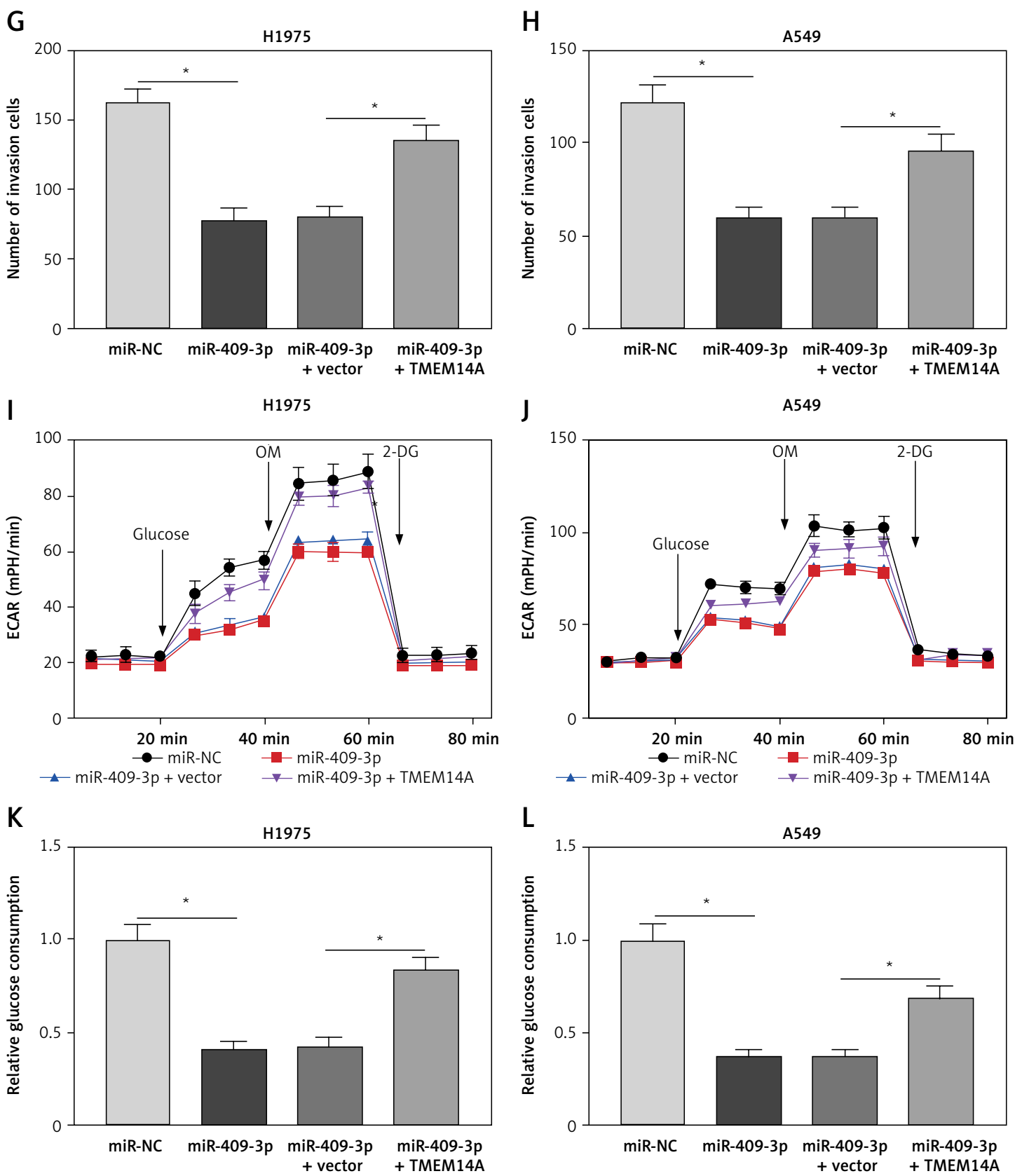

L
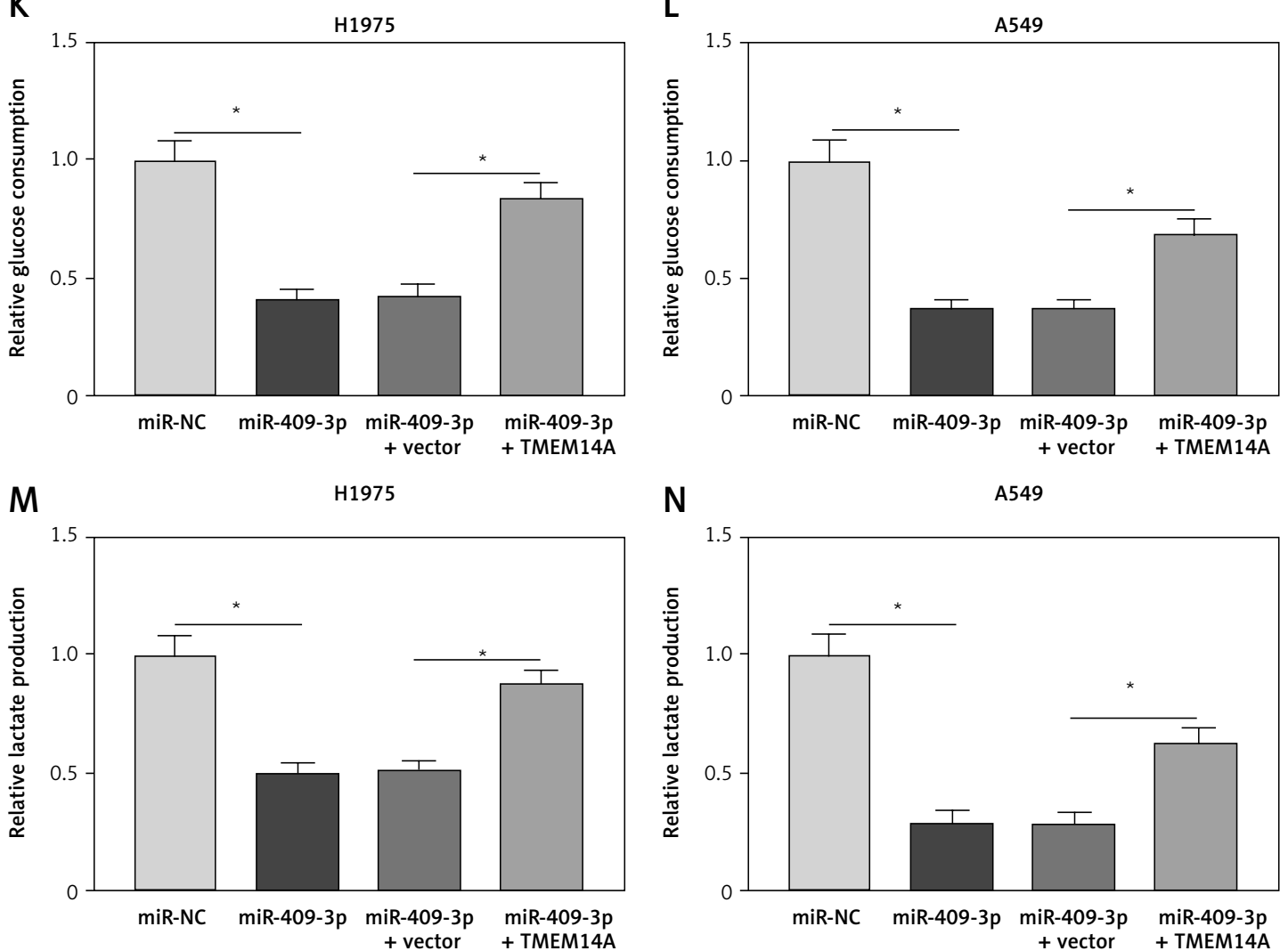

Figure 6. Cont. $\mathbf{E}-\mathbf{H}-$ The evaluation of cell migration $(\mathbf{E}, \mathbf{F})$ and invasion $(\mathbf{G}, \mathbf{H})$ was administered through the transwell assay. I-P - The assessment of glycolysis was performed by ECAR measurement (I, J), glucose consumption $(\mathbf{K}, \mathbf{L})$, lactate production $(\mathbf{M}, \mathbf{N})$ and the associated protein (GLUT1 and LDHA) examination via Western blot $(\mathbf{O}, \mathbf{P}) .{ }^{*} P<0.05$ 
0

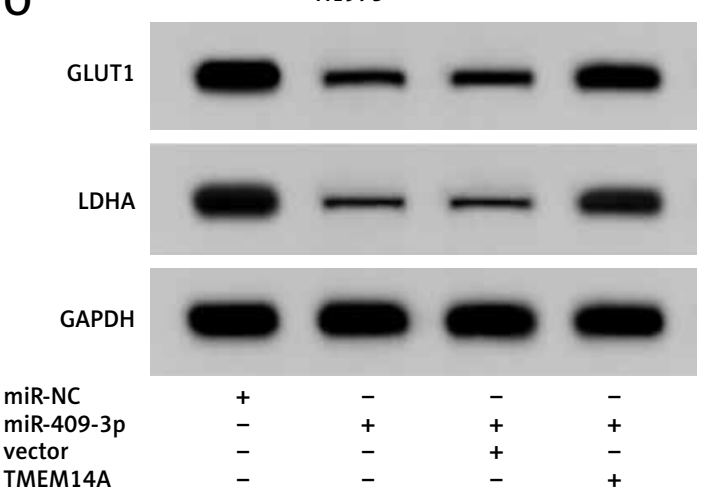

$\mathbf{P}$

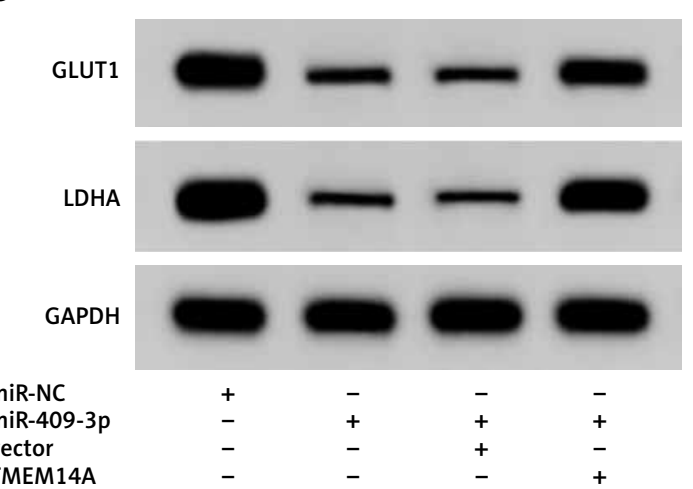

H1975

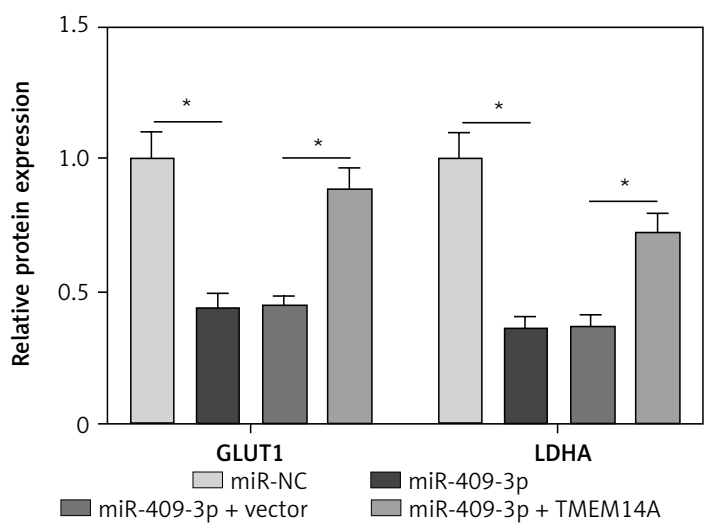

A549

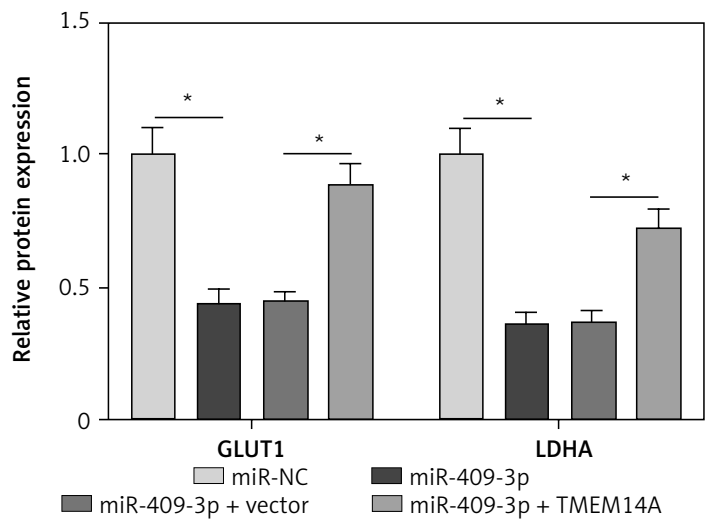

Figure 6. Cont. The associated protein (GLUT1 and LDHA) examination via Western blot $(\mathbf{0}, \mathbf{P}) .{ }^{\star} P<0.05$

(Figure $7 \mathrm{D}$ ) in excised tumors. Western blot indicated that the protein expression of TMEM14A was repressed by inhibiting circ_0001421 in vivo (Figure 7 E). Taken together, circ_0001421 knockdown inhibited tumor growth of NSCLC by regulating the miR-409-3p/TMEM14A axis in vivo.

\section{Discussion}

The molecular targeted therapy has been developed as a vital strategy to ameliorate the treatment and prognosis of NSCLC patients $[33,34]$. In the present study, we found the overexpression of circ_0001421 and TMEM14A as well as the down-regulation of miR-409-3p in NSCLC. Circ_0001421 could promote NSCLC development by acting as a miR-409-3p sponge to promote expression of TMEM14A, suggesting that circ_0001421 has the potential to become a molecular target in NSCLC treatment.

Currently, the circRNAs have drawn the attention of many researchers in NSCLC. CircRNA SMARCA5 repressed the proliferation, migration, and invasion in NSCLC cells via regulation of the miR-19b-3p/HOXA9 axis [35]. Hsa circ 0001946 had inhibitory effects on cell viability, migration and invasion of NSCLC through the nucleotide excision repair pathway [36]. Gao et al. attested that circ_0074027 elevated the proliferation and invasion abilities but reduced apoptosis by miR185-3p/BRD4/MADD signals in NSCLC [37], and circRNA ZFR improved the NSCLC progression by the miR-101-3p/CUL4B axis [38]. These studies implied different roles of circRNAs in NSCLC. Here, circ_0001421 was verified to be up-regulated in NSCLC, and knockdown of circ_0001421 exhibited the suppressive impacts on cell colony formation, migration and invasion. Glycolysis, a biochemically oxygen-independent metabolic pathway, can act as a target in tumor therapy [39, 40]. Circ_0001421 down-regulation led to inhibition of glycolysis (ECAR, glucose consumption, lactate production and GLUT1 and LDHA protein levels). The oncogenic role of circ_0001421 was ascertained in NSCLC for the first time.

Regarding the functional mechanism behind circ_0001421 in NSCLC, we found the miRNA target of circ_0001421 and validated that circ 0001421 targeted miR-409-3p. According to the previous studies, miR-409-3p plays an anticancer role in the majority of cancers. For instance, miR409-3p inhibited osteosarcoma cell proliferation and invasion via directly regulating ZEB1 [41]. Zhang et al. observed the down-regulation of miR- 
A

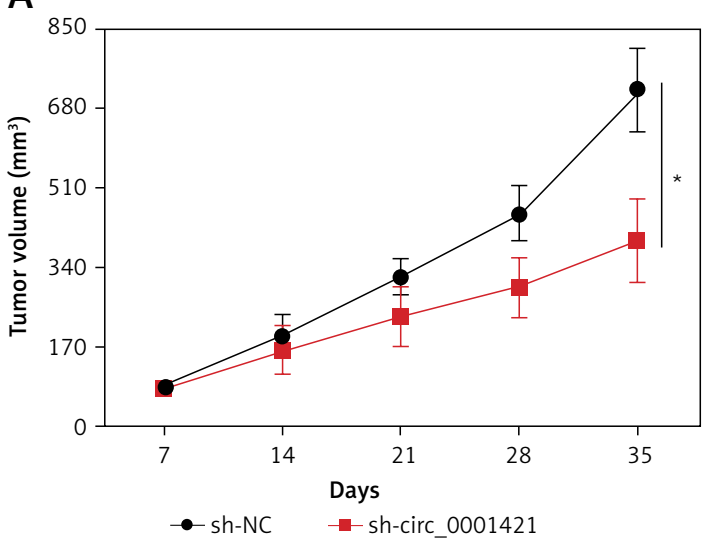

C
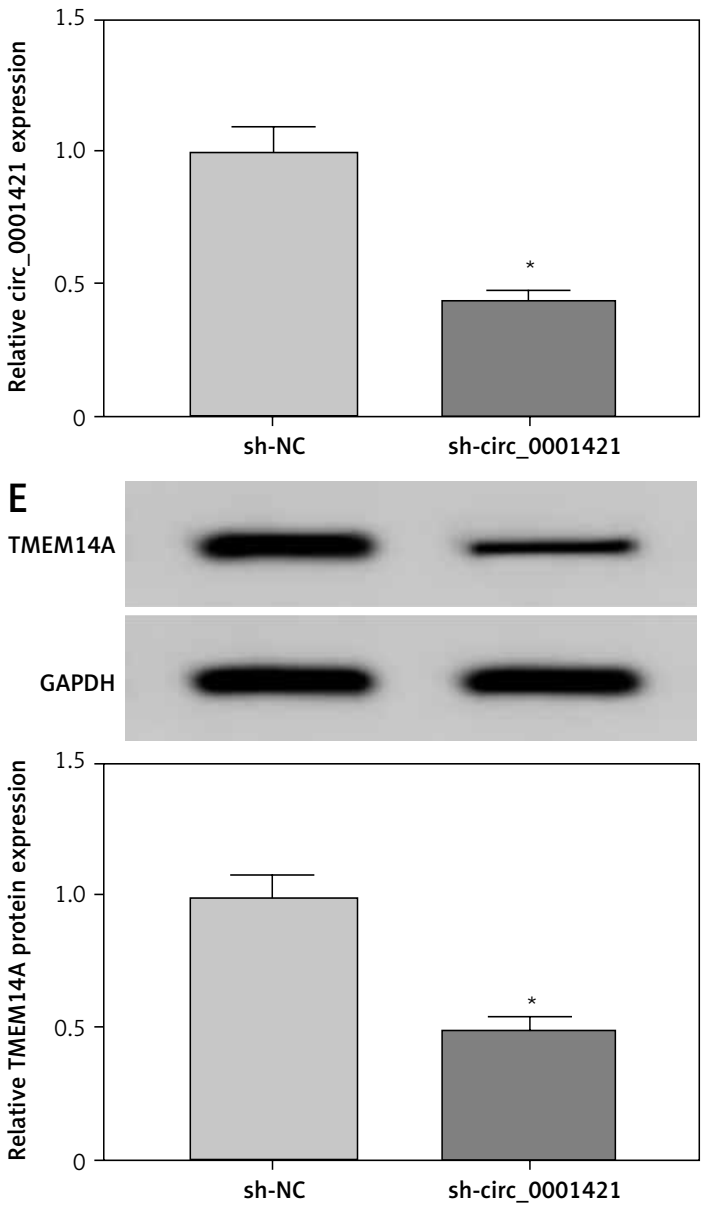

409-3p in breast cancer and miR-409-3p hindered cell growth and invasion via inhibiting Akt1 [42] In line with this, rescue assays demonstrated that miR-409-3p up-regulation was responsible for the suppression of circ_0001421 knockdown in NSCLC progression, suggesting the suppressive role of miR-409-3p in NSCLC. Moreover, TMEM14A was a downstream target of miR-409$3 p$ and miR-409-3p acted as the tumor inhibitor in NSCLC by targeting TMEM14A. Additionally, it is in teresting that circ_0001421 positively modulated TMEM14A expression by functioning as a sponge
B
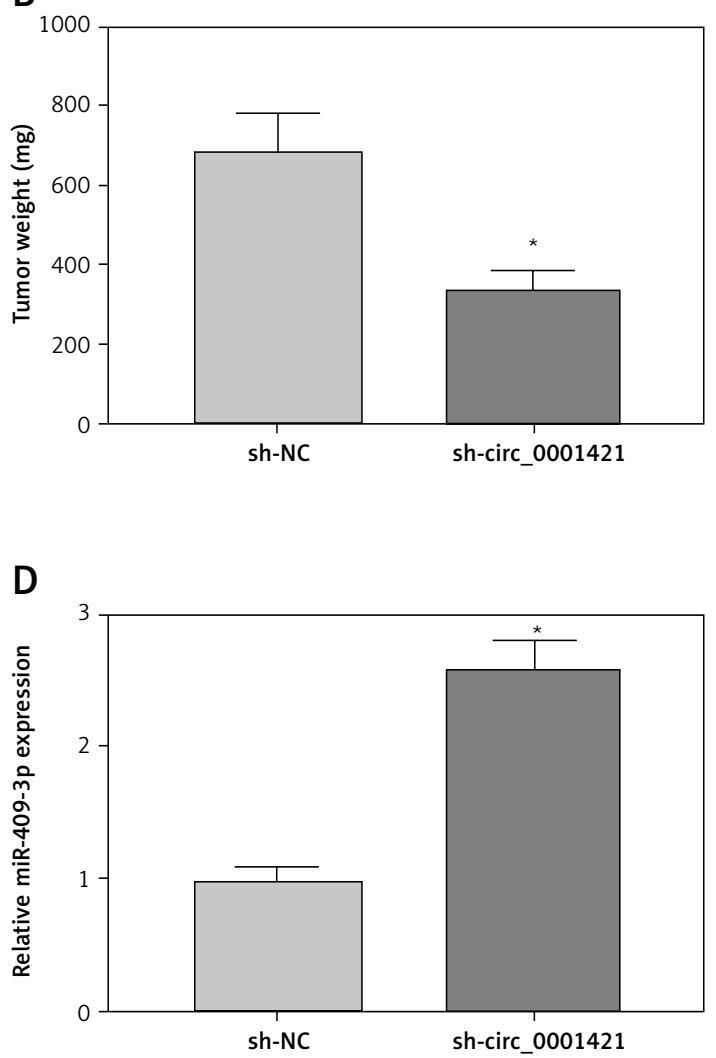

Figure 7. Depression of circ_0001421 reduced tumor growth of NSCLC via regulation of the miR409-3p/TMEM14A axis in vivo. A - Tumor volume was estimated weekly post-injection with A549 cells expressed sh-circ_0001421 or sh-NC. B - The weight of excised tumors was measured. C, D - The expression levels of circ 0001421 and miR-409$3 p$ were examined by $q R \bar{T}-P C R$ in tumors. $E$ - The protein level of TMEM14A was determined using Western blot. ${ }^{*} P<0.05$

of miR-409-3p. Judging from these results, we concluded that the effects of circ_0001421 on NSCLC cells were achieved by the miR-409-3p/TMEM14A axis. The circRNA-miRNA-mRNA regulatory network has been presented in multiple cancers [4345]. Our experiments in vivo also suggested that circ_0001421 enhanced tumor growth through the miR-409-3p/TMEM14A axis.

Altogether, this study showed that circ_0001421 supported cell colony formation, migration, invasion and glycolysis in NSCLC via regulating the miR-409-3p/TMEM14A axis. The oncogenic role 
of circ 0001421 in NSCLC was revealed for the first time and the circ 0001421/miR-409-3p/ TMEM14A axis afforded an unambiguous molecular pathomechanism for NSCLC, which might contribute to the understanding of the initiation and development of NSCLC in the circRNA field. Our findings indicated that circ 0001421 could improve the treatment of NSCLC as a novel molecular therapeutic target.

\section{Availability of data and materials}

The analyzed data sets generated during the present study are available from the corresponding author on reasonable request.

\section{Ethics approval and consent to participate}

The present study was approved by the ethical review committee of Xiantao First People's Hospital Affiliated to Changjiang University.

\section{Conflict of interest}

The authors declare no conflict of interest.

\section{References}

1. Bray F, Ferlay J, Soerjomataram I, Siegel RL, Torre LA, Jemal A. Global cancer statistics 2018: GLOBOCAN estimates of incidence and mortality worldwide for 36 cancers in 185 countries. CA Cancer J Clin 2018; 68: 394-424.

2. Gridelli C, Rossi A, Carbone DP, et al. Non-small-cell lung cancer. Nat Rev Dis Primers 2015; 1: 15009.

3. Nadal E, Massuti B, Domine M, Garcia-Campelo R, Cobo M, Felip E. Immunotherapy with checkpoint inhibitors in non-small cell lung cancer: insights from long-term survivors. Cancer Immunol Immunother 2019; 68: 341-52.

4. Masood A, Kancha RK, Subramanian J. Epidermal growth factor receptor (EGFR) tyrosine kinase inhibitors in nonsmall cell lung cancer harboring uncommon EGFR mutations: Focus on afatinib. Semin Oncol 2019; 46: 271-83.

5. Rosas G, Ruiz R, Araujo JM, Pinto JA, Mas L. ALK rearrangements: Biology, detection and opportunities of therapy in non-small cell lung cancer. Crit Rev Oncol Hematol 2019; 136: 48-55.

6. Arbour KC, Riely GJ. Systemic therapy for locally advanced and metastatic non-small cell lung cancer: a review. JAMA 2019; 322: 764-74.

7. Chen Y, Lu L, Feng B, et al. Non-coding RNAs as emerging regulators of epithelial to mesenchymal transition in non-small cell lung cancer. Oncotarget 2017; 8: 36787-99.

8. Wei MM, Zhou GB. Long non-coding RNAs and their roles in non-small-cell lung cancer. Genomics Proteomics Bioinformatics 2016; 14: 280-8.

9. Wilusz J E, Sharp P A. Molecular biology. A circuitous route to noncoding RNA. Science 2013; 340: 440-1.

10. Memczak S, Jens M, Elefsinioti $A$, et al. Circular RNAs are a large class of animal RNAs with regulatory potency. Nature 2013; 495: 333-8.

11. Guan X, Zong ZH, Liu Y, Chen S, Wang LL, Zhao Y. circPUM1 promotes tumorigenesis and progression of ovarian cancer by sponging miR-615-5p and miR-67535p. Mol Ther Nucleic Acids 2019; 18: 882-92.
12. Wang X, Wang X, Li W, Zhang Q, Chen J, Chen T. Up-regulation of hsa_circ_0000517 predicts adverse prognosis of hepatocellular carcinoma. Front Oncol 2019; 9: 1105.

13. Liu W, Ma W, Yuan Y, Zhang Y, Sun S. Circular RNA hsa circRNA_103809 promotes lung cancer progression via facilitating ZNF121-dependent MYC expression by sequestering miR-4302. Biochem Biophys Res Commun 2018; 500: 846-51.

14. Jin M, Shi C, Yang C, Liu J, Huang G. Upregulated circRNA ARHGAP10 predicts an unfavorable prognosis in NSCLC through regulation of the miR-150-5p/GLUT-1 axis. Mol Ther Nucleic Acids 2019; 18: 219-31.

15. Mendell JT, Olson EN. MicroRNAs in stress signaling and human disease. Cell 2012; 148: 1172-87.

16. Hammond SM. An overview of microRNAs. Adv Drug Deliv Rev 2015; 87: 3-14.

17. Yin Z, XU M, Li P. miRNA-221 acts as an oncogenic role by directly targeting TIMP2 in non-small-cell lung carcinoma. Gene 2017; 620: 46-53.

18. Ma HP, Kong WX, Li XY, Li W, Zhang Y, Wu Y. miRNA-223 is an anticancer gene in human non-small cell lung cancer through the PI3K/AKT pathway by targeting EGFR. Oncol Rep 2019; 41: 1549-59.

19. Song Q, Ji Q Xiao J, et al. miR-409 Inhibits human nonsmall-cell lung cancer progression by directly targeting SPIN1. Mol Ther Nucleic Acids 2018; 13: 154-63.

20. Qu R, Chen X, Zhang C. LncRNA ZEB1-AS1/miR-409-3p/ ZEB1 feedback loop is involved in the progression of non-small cell lung cancer. Biochem Biophys Res Commun 2018; 507: 450-6.

21. Zhang Q, Chen X, Zhang X, Zhan J, Chen J. Knockdown of TMEM14A expression by RNAi inhibits the proliferation and invasion of human ovarian cancer cells. Biosci Rep 2016; 36: e00298.

22. An J, Shi H, Zhang N, Song S. Elevation of circular RNA circ_0003645 forecasts unfavorable prognosis and facilitates cell progression via miR-1179/TMEM14A pathway in non-small cell lung cancer. Biochem Biophys Res Commun 2019; 511: 921-5.

23. Livak KJ, Schmittgen TD. Analysis of relative gene expression data using real-time quantitative PCR and the 2(-Delta Delta C(T)) method. Methods 2001; 25: 402-8.

24. Liu L, Wang Y, Bai R, Yang K, Tian Z. MiR-186 inhibited aerobic glycolysis in gastric cancer via HIF-1alpha regulation. Oncogenesis 2016; 5: e224.

25. Taylor SC, Berkelman T, Yadav G, Hammond M. A defined methodology for reliable quantification of Western blot data. Mol Biotechnol 2013; 55: 217-26.

26. Jiao L, Wang S, Zheng Y, et al. Betulinic acid suppresses breast cancer aerobic glycolysis via caveolin-1/NF-kappaB/c-Myc pathway. Biochem Pharmacol 2019; 161: $149-62$.

27. Brandi J, Cecconi D, Cordani M, et al. The antioxidant uncoupling protein 2 stimulates hnRNPA2/B1, GLUT1 and PKM2 expression and sensitizes pancreas cancer cells to glycolysis inhibition. Free Radic Biol Med 2016; 101: 305-16.

28. Chen L, Zhang S, Wu J, et al. circRNA_100290 plays a role in oral cancer by functioning as a sponge of the miR-29 family. Oncogene 2017; 36: 4551-61.

29. Zhao F, Chen CW, Yang WW, et al. Hsa circRNA 0059655 plays a role in salivary adenoid cystic carcinoma by functioning as a sponge of miR-338-3p. Cell Mol Biol (Noisy-le-grand) 2018; 64: 100-6.

30. Yang J, Cong X, Ren $M$, et al. Circular RNA hsa cirCRNA_0007334 is predicted to promote MMP7 and COL1A1 expression by functioning as a miRNA sponge in 
pancreatic ductal adenocarcinoma. J Oncol 2019; 2019: 7630894.

31. Hassanlou M, Soltani BM, Medlej A, Kay M, Mowla SJ. Hsa-miR-6165 downregulates insulin-like growth factor-1 receptor (IGF-1R) expression and enhances apoptosis in SW480 cells. Biol Chem 2020; 401: 477-85.

32. Pu M, Chen J, Tao Z, et al. Regulatory network of miRNA on its target: coordination between transcriptional and post-transcriptional regulation of gene expression. Cell Mol Life Sci 2019; 76: 441-51.

33. Fujimoto J, Wistuba II. Current concepts on the molecular pathology of non-small cell lung carcinoma. Semin Diagn Pathol 2014; 31: 306-13.

34. Prabhu VV, Devaraj N. Epidermal growth factor receptor tyrosine kinase: a potential target in treatment of nonsmall-cell lung carcinoma. J Environ Pathol Toxicol Oncol 2017; 36: 151-8

35. Wang Y, Li H, Lu H, Qin Y. Circular RNA SMARCA5 inhibits the proliferation, migration, and invasion of non-small cell lung cancer by miR-19b-3p/HOXA9 axis. Onco Targets Ther 2019; 12: 7055-65.

36. Huang MS, Liu JY, Xia XB, et al. Hsa_circ_0001946 inhibits lung cancer progression and mediates cisplatin sensitivity in non-small cell lung cancer via the nucleotide excision repair signaling pathway. Front Oncol 2019; 9: 508.

37. Gao P, Wang Z, Hu Z, Jiao X, Yao Y. Circular RNA circ_0074027 indicates a poor prognosis for NSCLC patients and modulates cell proliferation, apoptosis, and invasion via miR-185-3p mediated BRD4/MADD activation. J Cell Biochem 2020; 121: 2632-42.

38. Zhang H, Wang X, Hu B, Zhang F, Wei H, Li L. Circular RNA ZFR accelerates non-small cell lung cancer progression by acting as a miR-101-3p sponge to enhance CUL4B expression. Artif Cells Nanomed Biotechnol 2019; 47: 3410-6.

39. Ganapathy-Kanniappan S, Geschwind JF. Tumor glycolysis as a target for cancer therapy: progress and prospects. Mol Cancer 2013; 12: 152.

40. Ganapathy-Kanniappan S. Molecular intricacies of aerobic glycolysis in cancer: current insights into the classic metabolic phenotype. Crit Rev Biochem Mol Biol 2018; 53: 667-82.

41. Wu L, Zhang Y, Huang Z, et al. MiR-409-3p inhibits cell proliferation and invasion of osteosarcoma by targeting zinc-finger E-box-binding homeobox-1. Front Pharmacol 2019; 10: 137.

42. Zhang G, Liu Z, Xu H, Yang Q. miR-409-3p suppresses breast cancer cell growth and invasion by targeting Akt1. Biochem Biophys Res Commun 2016; 469: 189-95.

43. Xiao Y. Construction of a circRNA-miRNA-mRNA network to explore the pathogenesis and treatment of pancreatic ductal adenocarcinoma. J Cell Biochem 2020; 121 394-406.

44. Guan YJ, Ma JY, Song W. Identification of circRNA-miRNA-mRNA regulatory network in gastric cancer by analysis of microarray data. Cancer Cell Int 2019; 19: 183.

45. Liu K, Guo Y, Zheng K, et al. Identification of the circRNA-miRNA-mRNA regulatory network of Hsp90 inhibitor-induced cell death in colorectal cancer by integrated analysis. Gene 2020; 727: 144232. 